\title{
Hip Range of Motion Is Increased After Hip Arthroscopy for Femoroacetabular Impingement: A Systematic Review
}

\author{
David Filan, M.Sc., Karen Mullins, Ph.D., and Patrick Carton, M.D., F.R.C.S. (Orth.)
}

\begin{abstract}
Purpose: To investigate the impact of arthroscopic correction of symptomatic femoroacetabular impingement on postoperative hip range of motion (ROM), as an objectively measured postoperative clinically reported outcome. Methods: A systematic review of the current literature was performed according to the Preferred Reporting Items for Systematic Reviews and Meta-Analyses (PRISMA) guidelines. PubMed, OVID/MEDLINE, EMBASE, and Cochrane databases were queried in November 2020. Studies not reporting pre- to postoperative ROM measurements were excluded. Methodologic quality was assessed using the MINORS assessment, and certainty of evidence was assessed using the GRADE approach. Effect size using standardized mean differences assessed magnitude of change between pre- and postoperative ROM. Results: In total, 23 studies were included evaluating 2,332 patients. Mean age ranged from 18 to 44.2 years. Flexion, internal rotation (IR), and external rotation (ER) were the predominantly measured ROMs reported in $91 \%$, $100 \%$ and $65 \%$ of studies, respectively. Observed change following hip arthroscopy was considered significant in 57.1\% (flexion), $74 \%$ (IR), and $20 \%$ (ER). Effect size of change in significantly improved ROMs were weak (16.7\% flexion, 33.3\% ER), moderate (58.3\% flexion, 29.4\% IR), and large (25\% flexion, 64.7\% IR, 66.7\% ER). For goniometric assessment mean observed changes ranged as follows: flexion: $0.1^{\circ}$ to $12.2^{\circ}$; IR: $3.6^{\circ}$ to $21.9^{\circ}$; ER: $-2.6^{\circ}$ to $12.8^{\circ}$. For computed $^{\circ}$ tomography-simulated assessment, the mean observed change ranged as follows: flexion: $3.0^{\circ}$ to $8.0^{\circ}$; IR $9.3^{\circ}$ to $14.0^{\circ}$. Conclusions: Outcome studies demonstrate overall increased range of flexion and IR post-hip arthroscopy, with a moderate and large effect respectively. Change in ER is less impacted following hip arthroscopy. Certainty of evidence to support this observation is low. Current research evaluating changes in this functional ability is limited by a lack of prospective studies and non-standardized measurement evaluation techniques. Level of Evidence: Level IV, systematic review of Level II-IV studies.
\end{abstract}

$\mathbf{F}$ emoroacetabular impingement (FAI) is primarily considered a mechanical, motion-related hip disorder, exacerbated by abnormal bony morphology of the acetabulum (overcoverage, pincer deformity), femur (asphericity, cam deformity) or both (mixed impingement).

Progressive in their development, these bony prominences in an otherwise-healthy hip can be considered

From the Hip and Groin Clinic (P.C.), UPMC Whitfield (D.F., K.M., P.C.), Waterford, Ireland.

The authors report that they have no conflicts of interest in the authorship and publication of this article. Full ICMJE author disclosure forms are available for this article online, as supplementary material.

Received July 27, 2021; accepted December 8, 2021.

Address correspondence to David Filan, Suite 5, UPMC Whitfield, Butlerstown North, Cork Road, Waterford, Ireland.E-mail: filand@upmc.ie

(C) 2022 THE AUTHORS. Published by Elsevier Inc. on behalf of the Arthroscopy Association of North America. This is an open access article under the CC BY-NC-ND license (http://creativecommons.org/licenses/by-nc-nd/4.0/). 2666-061X/211040

https://doi.org/10.1016/j.asmr.2021.12.001 analogous to mechanical blocks to end range of motion (ROM). Clinical examination typically reproduces pain upon specific patterns of hip movement: flexion, adduction and internal rotation (FADIR, impingement test), and/or flexion, abduction, and external rotation (FABER test). ${ }^{1-3}$ Additional diagnostic workup includes a loss or marked reduction in hip ROM between the affected and unaffected contralateral joint, ${ }^{2,4-6}$ or in the case in which symptoms are experienced bilaterally, significant restriction in the available ROM from the acceptable normative ranges. ${ }^{7}$ Owing to the typical location of these deformities, movements such as flexion, adduction, and internal rotation ${ }^{8-13}$ often are reduced with abduction and external rotation being spared. ${ }^{11,12}$ While some previous studies have indicated reduced ROM in the presence of FAI-related morphology compared with controls without such deformities, ${ }^{14-16}$ the available evidence is conflicting. ${ }^{17}$

Hip arthroscopy (HA) is an effective and increasingly performed surgical intervention established in 
alleviating associated pain and functional disabilities for patients with symptomatic FAI. As part of this surgical correction, a primary focus is the removal of contributory abnormal bone, to reshape and restore the hip's normal anatomy and movement pattern, free from impingement. However, the literature describing the mechanical impact of this surgery on ROM is a less frequently reported clinical outcome overall, despite this being a consideration in resolving the pathology. Consequently, evidence-based normative values and scope for improvement in ROM following HA for FAI is under-reported and in stark contrast to the abundance of patient-reported outcome (PRO) studies available. $^{18,19}$

The purpose of this systematic review was to investigate the impact of arthroscopic correction of symptomatic FAI on postoperative hip ROM, as an objectively measured postoperative clinically reported outcome. We hypothesize that measurable ROM would be significantly improved following HA for FAI.

\section{Methods}

\section{Search Strategy}

This systematic review was performed in accordance with 2020 Preferred Reporting Items for Systematic Review and Meta-Analysis (PRISMA) guidelines. ${ }^{20}$ Our research question was established a priori as "What are the quantifiable changes to hip range of motion following the arthroscopic correction of femoroacetabular impingement?"

In accordance with PICO framework, our population of interest included human cases diagnosed with FAI, the intervention was hip arthroscopy, there was no comparison control group (i.e., exclusively assessing for those with diagnosed FAI undergoing arthroscopic treatment), and the outcome of interest was hip joint ROM. Electronic searches for all eligible studies was performed within each of the following databases: PubMed, OVID/MEDLINE, EMBASE, and the Cochrane database (Appendix Table l, available at www. arthroscopyjournal.org). The search query was performed November 2020 independently by 2 reviewers (D.F. and K.M.).

\section{Eligibility Criteria}

Inclusion criteria consisted of original studies involving patients $>16$ years of age, no upper age limit, undergoing arthroscopic FAI-corrective surgery, reporting a change in ROM as a clinical outcome and published in English. Exclusion criteria were (1) review articles/systematic reviews/meta-analyses/basic science/surgical techniques/letter to editor/narrative review/animal studies/cadaveric studies/conference abstracts/case reports; (2) surgical procedures other than HA; (3) hip pathology other than true FAI (e.g., patients with hip dysplasia, slipped capital femoral epiphysis, Legg-Calve-Perthes disease, avascular necrosis, osteoarthritis; (4) studies only reporting preoperative ROM; and (5) studies involving revision cases or where a concomitant pathology also was addressed.

\section{Study Selection}

Two reviewers independently screened articles identified following database query using the inclusion and exclusion criteria mentioned. Studies that did not adequately report relevant information in the title or abstract were selected for full text review. Following full-text review, studies that met the eligibility criteria were included for systematic review. Any discrepancy between reviewers during the search process was discussed in a consensus meeting with the assistance of a third reviewer (P.C.).

\section{Quality Assessment}

Two experienced clinical researchers (D.F., K.M.) independently assessed the methodologic quality of included studies. The validated MINORS (methodologic items for nonrandomized studies) criteria was used for quality assessment, ${ }^{21}$ which assess 8 critical aspects of study design for noncomparative clinical studies and an additional 4 aspects of study design for comparative clinical studies. Each item is given a score of 0 if information is not reported, 1 if information is reported but inadequate, and 2 if information is reported and adequate. The maximum possible score is 16 for noncomparative studies and 24 for comparative studies. For noncomparative studies, quality is assessed as follows: 0-4 very low quality; 5-8 low quality; 9-12 fair quality; and 13-16 high quality. For comparative studies quality is assessed as follows: 0-6 very low quality; 7-12 low quality; 13-18 fair quality; and 19-24 high quality. ${ }^{22}$ Any disagreements in overall rating were resolved by a third reviewer (P.C.). A kappa statistic was used to evaluate the level of interrater agreement with agreement classified a priori as: $<0.2$ poor; 0.21-0.4 fair; 0.41-0.60 moderate; 0.61-0.80 good; and 0.81-1.00 very good. Certainty of evidence was assessed using the Grades of Recommendation, Assessment, Development and Evaluation (GRADEpro GDT, McMaster University, 2020).

\section{Data Extraction}

Study identifiers (title, year, author, journal), study design, patient demographics (number of included hips, sex, age), follow-up duration, pre- and postoperative ROM details, technique used to evaluate ROM, pre- and postoperative radiographic measurements 
Fig 1. Preferred Reporting Items for Systematic Review and MetaAnalysis (PRISMA) flowchart for included studies. (FAI, femoroacetabular impingement; HA, hip arthroscopy; MRA, magnetic resonance arthroscopy; MRI, magnetic resonance imaging; $\mathrm{OA}$, osteoarthritis; ROM, range of motion.)

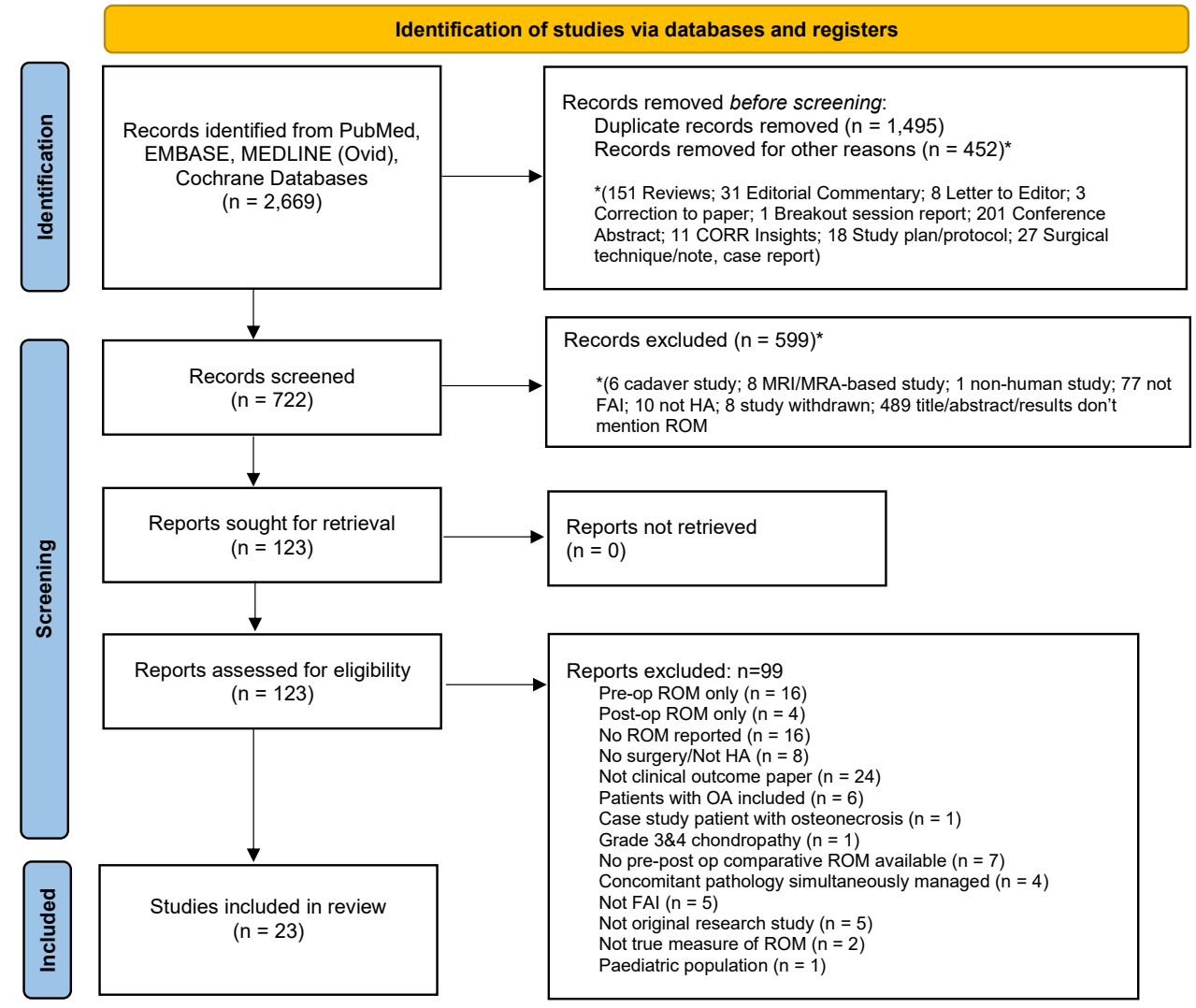

(alpha angle and lateral center edge angle [LCEA]) were extracted from the included studies by one reviewer (D.F.).

The primary outcome measure of interest for this review was a ROM in any plane.

To illustrate the change from preoperative to postoperative ROM scores, while accommodating variability within studies, a standardized mean difference (SMD) was calculated to estimate effect size using Cochrane Review Manager (RevMan v.5,4). If no standard deviation (SD) or standard error (SE) was reported by the authors, and a range of scores was given, the SD was estimated by dividing the range of scores by $6,{ }^{23}$ and the SMD was then calculated. If no range, SD or SE was given, the SMD was estimated using the sample size and $P$ value of the $t$-test used in the study. We calculated $95 \%$ confidence intervals (CIs) assuming normal distribution using the formula SMD $\pm 1.96 \times$ SE of the SMD. ${ }^{24}$ Cohen criteria were used to interpret individual study SMD where a large effect size was interpreted as SMD $\geq 0.8$, a moderate effect size $>0.5$ and $<0.8$, and a weak effect size $\leq 0.5$ and $\geq 0.2$. Forest plots using generic inverse variance data type were produced in RevMan to facilitate the interpretation of mean differences and 95\% CIs wherein the same system of units and measurement technique was used.

\section{Results}

\section{Study Characteristics/Demographics}

Twenty-three articles (Fig 1), evaluating 2,332 patients having undergone HA for FAI were included. The number of patients per study ranged from 10 to 688 with 11 studies evaluating unilaterally operated patients, 10 studies including at least one bilaterally operated patient in the cohort, and the remaining 2 studies not distinguishing between hips/patients. The mean age at time of surgery ranged from 18.0 to 44.2 years. One study ${ }^{25}$ did not report age. The proportion of female patients ranged from $0 \%$ to $100 \%$, with 11 studies having a predominantly female cohort, 9 predominantly male cohort, 1 had equal male-to-female cohort, and 2 did not distinguish. Follow-up time for the purpose of each study's primary outcome ranged from the day of surgery (ROM assessed intraoperatively) to a mean of 31.3 months (range 23.1-67.3 months).

\section{Methodologic Quality}

There were 3 Level II studies, 9 Level III studies, and 11 Level IV studies included. ${ }^{26}$

MINORS assessment rated the quality of the included studies as fair in $74 \%(17 / 23)$, high in $13 \%(3 / 23)$, low in $9 \%(2 / 23)$, and very low in $4 \%(1 / 23)$ (Table 1$)$. The 


\begin{tabular}{|c|c|c|c|c|c|c|c|c|c|c|}
\hline Author, year & $\begin{array}{l}\text { Level of } \\
\text { Evidence }\end{array}$ & Journal & Population & $\begin{array}{l}\text { Study Size } \\
\text { (Patients) }\end{array}$ & $\begin{array}{c}\text { Study } \\
\text { Size (Hips) }\end{array}$ & $\operatorname{Sex}(F / M)$ & Mean Age, y & Follow-up ${ }^{*}$ & $\begin{array}{c}\text { MINORS Quality } \\
\text { Rating }\end{array}$ & Study Purpose \\
\hline $\begin{array}{l}\text { Keating et al., } \\
2020^{39}\end{array}$ & IV & JHPS & $\begin{array}{l}\text { FAIS patients } \\
\text { participating in } \\
\text { Pilates }\end{array}$ & 22 & 22 & $22 / 0$ & $38.1 \pm 10.8$ & Minimum $24 \mathrm{mo}$ & Fair (10/16) & $\begin{array}{l}\text { (i) evaluate patients' ability to, } \\
\text { and rate of, return to Pilates } \\
\text { after hip arthroscopy for } \\
\text { FAIS and (ii) assess } \\
\text { postoperative performance } \\
\text { and weekly involvement } \\
\text { compared with preinjury } \\
\text { participation }\end{array}$ \\
\hline $\begin{array}{l}\text { Ragab et al., } \\
2018^{46}\end{array}$ & IV & $\begin{array}{l}\text { Alexandria } \\
\text { Journal of } \\
\text { Medicine }\end{array}$ & FAI patients & 40 & 40 & $20 / 20$ & $38.6 \pm 11.1$ & $\begin{array}{r}12.5 \pm 4.7 \mathrm{mo} \\
\quad(\text { range } 6-24)\end{array}$ & Very low $(4 / 16)$ & $\begin{array}{l}\text { To assess the results of } \\
\text { arthroscopic treatment of } \\
\text { FAI }\end{array}$ \\
\hline $\begin{array}{l}\text { Waterman } \\
\text { et al., } \\
2018^{43}\end{array}$ & IV & Arthroscopy & Golfers with FAIS & 29 & 31 & $6 / 23$ & $36.0 \pm 11.9$ & Minimum 2 y & Fair $(10 / 16)$ & $\begin{array}{l}\text { (i) Investigate whether patients } \\
\text { who reported playing golf } \\
\text { before arthroscopic hip } \\
\text { surgery for FAIS were able } \\
\text { to return to playing golf } \\
\text { postoperatively. (ii) To } \\
\text { determine whether hip } \\
\text { range of motion was } \\
\text { associated with } \\
\text { improvement in PROs and } \\
\text { golf-specific metrics }\end{array}$ \\
\hline $\begin{array}{l}\text { Flores et al., } \\
2018^{44}\end{array}$ & II & OJSM & FAI patients & 58 & 60 & $\begin{array}{l}\text { Early: } 15 / 15 \\
\text { Late: } 13 / 17\end{array}$ & $\begin{array}{l}\text { Early: } 37.2 \pm 11.5 \\
\quad \text { Late: } 35.3 \pm \\
10.8\end{array}$ & $\begin{array}{l}\text { Early: } 15.5 \pm 4.7 \\
\quad \text { mo } \\
\quad \text { Late: } 13.1 \pm 2.7 \\
\quad \text { mo }\end{array}$ & High (13/16) & $\begin{array}{l}\text { To evaluate the relationship } \\
\text { between surgeon } \\
\text { experience and patient } \\
\text { outcomes for the } \\
\text { arthroscopic treatment of } \\
\text { FAI. Primary outcome } \\
\text { measures were PRO scores, } \\
\text { secondary outcomes } \\
\text { included operation times } \\
\text { and complication rates }\end{array}$ \\
\hline $\begin{array}{l}\text { Carton and } \\
\text { Filan, } \\
2020^{27}\end{array}$ & III & OJSM & Athletes with FAI & 429 & 576 & $\begin{array}{l}\text { 23/553 (hips) } \\
21 / 408 \\
\text { (patients) }\end{array}$ & $25.9 \pm 9.7$ & $\begin{array}{l}2.4 \pm 0.7 y \text { (range } \\
2.0-2.5)\end{array}$ & Fair $(10 / 16)$ & $\begin{array}{l}\text { (i) Define the MCID at } 2 \text { years } \\
\text { postoperatively in } \\
\text { competitive athletes } \\
\text { undergoing hip arthroscopy } \\
\text { for symptomatic sports- } \\
\text { related FAI using existing } \\
\text { anchor- and distribution- } \\
\text { based methods; (ii) derive a } \\
\text { measure of the MCID using } \\
\text { the percentage of possible } \\
\text { improvement method and } \\
\text { compare against existing } \\
\text { techniques }\end{array}$ \\
\hline
\end{tabular}




\begin{tabular}{|c|c|c|c|c|c|c|c|c|c|c|}
\hline Author, year & $\begin{array}{l}\text { Level of } \\
\text { Evidence }\end{array}$ & Journal & Population & $\begin{array}{l}\text { Study Size } \\
\text { (Patients) }\end{array}$ & $\begin{array}{c}\text { Study } \\
\text { Size (Hips) }\end{array}$ & $\operatorname{Sex}(\mathrm{F} / \mathrm{M})$ & Mean Age, y & Follow-up ${ }^{*}$ & $\begin{array}{l}\text { MINORS Quality } \\
\text { Rating }\end{array}$ & Study Purpose \\
\hline $\begin{array}{l}\text { Stone et al., } \\
2019^{29}\end{array}$ & III & JHPS & $\begin{array}{l}\text { FAIS patients (with } \\
\text { and without } \\
\text { GJL) }\end{array}$ & $\begin{array}{l}125 \\
(\mathrm{NGJL}=100 \\
\mathrm{GJL}=25)\end{array}$ & $\begin{array}{c}125(100 \\
25)\end{array}$ & $\begin{array}{l}\text { NGJL: } 100 / 0 \\
\text { GJL: } 25 / 0\end{array}$ & $\begin{array}{l}\text { NGJL: } \\
22.7 \pm 8.7 \\
\text { GJL: } \\
18 \pm 6.3\end{array}$ & $29.3 \pm 8.0 \mathrm{mo}$ & Fair $(17 / 24)$ & $\begin{array}{l}\text { To evaluate the postoperative } \\
\text { clinical and functional } \\
\text { outcomes in patients with } \\
\text { and without generalized } \\
\text { joint laxity following hip } \\
\text { arthroscopy for FAIS and } \\
\text { capsular plication }\end{array}$ \\
\hline $\begin{array}{l}\text { Ross et al., } \\
2018^{36}\end{array}$ & II & HSS Journal & $\begin{array}{l}\text { American football } \\
\text { linesmen }\end{array}$ & 13 & 17 & $0 / 13$ & $24.7 \pm 4.0$ & $\mathrm{n} / \mathrm{a}$ & Fair (11/16) & $\begin{array}{l}\text { (i) to characterize the } \\
\text { radiographic deformity and } \\
\text { dynamic impingement } \\
\text { observed in a consecutive } \\
\text { series of American football } \\
\text { linesmen with symptomatic, } \\
\text { mechanical hip pain who } \\
\text { underwent surgical } \\
\text { treatment for FAI; (ii) to use } \\
\text { software analysis to identify } \\
\text { the location of impingement } \\
\text { and terminal range of } \\
\text { motion and the effects of } \\
\text { simulated correction }\end{array}$ \\
\hline $\begin{array}{l}\text { Polesello et al., } \\
2009^{48}\end{array}$ & IV & $\begin{array}{l}\text { Revista } \\
\quad \text { Brasileria de } \\
\text { Orthopaedia }\end{array}$ & FAI patients & 28 & - & $9 / 19$ & 34 & $\begin{array}{l}27 \text { mo (range 12- } \\
60 \text { ) }\end{array}$ & Low $(7 / 16)$ & $\begin{array}{l}\text { To assess the short-term results } \\
\text { of the arthroscopic } \\
\text { treatment of FAI }\end{array}$ \\
\hline $\begin{array}{l}\text { Mullins et al., } \\
2020^{28}\end{array}$ & II & KSSTA & $\begin{array}{l}\text { Athletes with FAI } \\
\text { vs control }\end{array}$ & 47 (32 controls) & 70 hips & $0 / 47$ & $24.6 \pm 4.8$ & 1 year & High $(19 / 24)$ & $\begin{array}{l}\text { To measure the changes in } \\
\text { athletic performance in } \\
\text { athletes treated } \\
\text { arthroscopically for FAI and } \\
\text { compare results to a } \\
\text { matched controlled athletic } \\
\text { cohort, over a 1-year period }\end{array}$ \\
\hline $\begin{array}{l}\text { Stone et al., } \\
2019^{42}\end{array}$ & III & AJSM & FAIS patients & $\begin{array}{l}688 \\
\text { Nonpersistent, } \\
\text { 514; persistent } \\
\text { 174) }\end{array}$ & $\begin{array}{l}688 \\
\text { Nonpersistent, } \\
\text { 514; persistent } \\
\text { 174) }\end{array}$ & $\begin{array}{l}\text { Nonpersistent } \\
\quad 334 / 180 \\
\text { Persistent 115/ } \\
59\end{array}$ & $\begin{array}{l}\text { Nonpersistent } \\
\quad 32.4 \pm 12.6 \\
\text { Persistent group } \\
35.9 \pm 12.2\end{array}$ & Min 2 years & Fair (17/24) & $\begin{array}{l}\text { To identify patient } \\
\text { characteristics that predict } \\
\text { persistent postoperative pain } \\
\text { and function among people } \\
\text { undergoing hip arthroscopy } \\
\text { for FAIS }\end{array}$ \\
\hline $\begin{array}{l}\text { Frank et al., } \\
2018^{38}\end{array}$ & IV & Sports $\theta$ Health & $\begin{array}{l}\text { FAIS patients } \\
\text { participating in } \\
\text { yoga }\end{array}$ & 42 & 45 & $38 / 4$ & $35 \pm 9$ & $\begin{array}{l}30.5 \pm 12.0 \mathrm{mo} \\
\quad \text { (range } 12-44 \\
\text { mo) }\end{array}$ & Fair $(12 / 16)$ & $\begin{array}{l}\text { To evaluate patients' ability to } \\
\text { return to yoga after hip } \\
\text { arthroscopy for FAIS }\end{array}$ \\
\hline $\begin{array}{l}\text { Frank et al., } \\
2018^{45}\end{array}$ & IV & Sports $\theta$ Health & $\begin{array}{l}\text { FAIS patients } \\
\text { participating in } \\
\text { cycling }\end{array}$ & 58 patients & 60 hips & $36 / 22$ & $30.0 \pm 7.1$ & $31.1 \pm 0.7 \mathrm{mo}$ & Fair (11/16) & $\begin{array}{l}\text { To evaluate patients' ability to } \\
\text { return to cycling after hip } \\
\text { arthroscopy for FAIS }\end{array}$ \\
\hline
\end{tabular}




\begin{tabular}{|c|c|c|c|c|c|c|c|c|c|c|}
\hline Author, year & $\begin{array}{l}\text { Level of } \\
\text { Evidence }\end{array}$ & Journal & Population & $\begin{array}{l}\text { Study Size } \\
\text { (Patients) }\end{array}$ & $\begin{array}{c}\text { Study } \\
\text { Size (Hips) }\end{array}$ & $\operatorname{Sex}(F / M)$ & Mean Age, y & Follow-up ${ }^{*}$ & $\begin{array}{l}\text { MINORS Quality } \\
\text { Rating }\end{array}$ & Study Purpose \\
\hline $\begin{array}{l}\text { Levy et al., } \\
2017^{40}\end{array}$ & III & $A J S M$ & FAI patients & $\begin{array}{l}\text { Atypical: } \\
28 \\
\text { Typical: } \\
56\end{array}$ & - & $\begin{array}{l}\text { Atypical } \\
18 / 10 \\
\text { Typical } \\
36 / 20\end{array}$ & $\begin{array}{l}\text { All: } 35.4 \pm 9.8 \\
\quad \text { Atypical: } 35.8 \pm \\
9.9 \\
\text { Typical: } 35.2 \pm \\
9.9\end{array}$ & $2.6 \pm 0.6 \mathrm{y}$ & Fair $(17 / 24)$ & $\begin{array}{l}\text { To compare outcomes of hip } \\
\text { arthroscopy for FAI in } \\
\text { patients who experience } \\
\text { atypical posterior pain } \\
\text { versus a matched control } \\
\text { group who report the typical } \\
\text { anterior groin pain } \\
\text { presentation }\end{array}$ \\
\hline $\begin{array}{l}\text { Nawabi et al., } \\
2016^{41}\end{array}$ & III & AJSM & $\begin{array}{l}\text { FAI patients (BD } \\
\text { versus no } \\
\text { dysplasia) }\end{array}$ & $\begin{array}{l}\text { BD Group: } \\
46 \\
\text { Control: } \\
131\end{array}$ & $\begin{array}{l}\text { BD group: } \\
55 \\
\text { Control: } \\
152\end{array}$ & $\begin{array}{l}\text { BD Group: } \\
22 / 24 \\
\text { Control: } \\
73 / 58\end{array}$ & $\begin{array}{l}\text { BD Group: } \\
29.8 \pm 9.4 \\
\text { Control: } \\
29.6 \pm 10.3\end{array}$ & $\begin{array}{l}31.3 \pm 7.6 \text { months } \\
\text { (range 23.1- } \\
67.3)- \\
\text { unrevised } \\
\text { patients. } \\
21.6 \pm 13.3 \\
\text { (range 4.7-40.6) } \\
\text { - revised } \\
\text { patients }\end{array}$ & Fair (17/24) & $\begin{array}{l}\text { To compare outcomes after hip } \\
\text { arthroscopy for FAI in } \\
\text { patients with BD compared } \\
\text { with a control group of } \\
\text { patients without BD. Focus } \\
\text { on PROMs and reoperation } \\
\text { rates }\end{array}$ \\
\hline $\begin{array}{l}\text { Fabricant et al., } \\
2015^{31}\end{array}$ & III & $J B J S(A m)$ & FAI patients & $\begin{array}{l}\text { All: } 243 \\
\text { Decreased: } \\
37 \\
\text { Normal: } \\
149 \\
\text { Increased: } \\
57\end{array}$ & - & $\begin{array}{l}\text { Total: } 123 / 120 \\
\text { Decreased: } \\
41 \% / 59 \% \\
\text { Normal: } \\
50 \% / 50 \% \\
\text { Increased: } \\
58 \% / 42 \%\end{array}$ & $\begin{array}{l}\text { All: } 29.2 \mathrm{y} \\
\text { Decreased: } 28 \pm \\
9 \\
\text { Normal: } \\
30 \pm 11 \\
\text { Increased: } 29 \pm \\
10\end{array}$ & $\begin{array}{l}21 \text { mo (range 12- } \\
42 \text { ) }\end{array}$ & Fair $(12 / 16)$ & $\begin{array}{l}\text { To (i) investigate the } \\
\text { association between } \\
\text { proximal femoral version } \\
\text { and disease-specific, patient- } \\
\text { reported clinical outcomes } \\
\text { following arthroscopic } \\
\text { decompression of FAI; (ii) to } \\
\text { investigate associations of } \\
\text { combined femoral and } \\
\text { acetabular version (the } \\
\text { McKibbin index) with } \\
\text { patient-reported outcomes. }\end{array}$ \\
\hline $\begin{array}{l}\text { Ross et al., } \\
2015^{37}\end{array}$ & III & CORR & FAI patients & $\begin{array}{l}\text { Revision group: } \\
\quad 47 \\
\text { Successful } \\
\text { group: } \\
65\end{array}$ & $\begin{array}{l}\text { Revision group: } \\
\quad 50 \\
\text { Successful } \\
\text { group: } \\
65\end{array}$ & $\begin{array}{l}\text { Revision group: } \\
27 / 23 \\
\text { Successful } \\
\text { group: } \\
37 / 28\end{array}$ & $\begin{array}{l}\text { Revision group: } \\
29 \pm 9 \\
\text { Successful } \\
\text { group: } \\
25 \pm 9\end{array}$ & $\mathrm{n} / \mathrm{a}$ & Fair $(12 / 16)$ & $\begin{array}{l}\text { To (i) define the 3D } \\
\text { morphology of hips with } \\
\text { residual pain and/or } \\
\text { restricted ROM after } \\
\text { corrective arthroscopic FAI } \\
\text { surgery before revision } \\
\text { surgery; (ii) determine the } \\
\text { residual limitation in ROM } \\
\text { in these patients using } \\
\text { dynamic, computer-assisted, } \\
\text { 3D analysis; (iii) compare } \\
\text { the 3D morphology of hips } \\
\text { undergoing revision FAI } \\
\text { surgery with post-operative } \\
\text { 3D morphology of hips that } \\
\text { underwent successful } \\
\text { primary surgical treatment }\end{array}$ \\
\hline
\end{tabular}




\begin{tabular}{|c|c|c|c|c|c|c|c|c|c|c|}
\hline Author, year & $\begin{array}{l}\text { Level of } \\
\text { Evidence }\end{array}$ & Journal & Population & $\begin{array}{l}\text { Study Size } \\
\text { (Patients) }\end{array}$ & $\begin{array}{c}\text { Study } \\
\text { Size (Hips) }\end{array}$ & $\operatorname{Sex}(F / M)$ & Mean Age, y & Follow-up * & $\begin{array}{l}\text { MINORS Quality } \\
\text { Rating }\end{array}$ & Study Purpose \\
\hline $\begin{array}{l}\text { Stähelin et al., } \\
2008^{47}\end{array}$ & IV & Arthroscopy & $\begin{array}{l}\text { FAI patients } \\
\text { (specifically cam } \\
\text { impingement) }\end{array}$ & 14 & 14 & $6 / 8$ & $41.8 \pm 13.8$ & $6 \mathrm{mo}$ & Fair (10/16) & $\begin{array}{l}\text { To determine the accuracy of } \\
\text { arthroscopic restoration of } \\
\text { femoral offset as well as the } \\
\text { early clinical outcomes of } \\
\text { arthroscopic debridement } \\
\text { and femoral offset } \\
\text { restoration and whether } \\
\text { there is a correlation } \\
\text { between accuracy and } \\
\text { outcome. }\end{array}$ \\
\hline $\begin{array}{l}\text { Riff et al., } \\
2018^{34}\end{array}$ & IV & AJSM & HIIT athletes & 32 & 37 & $19 / 13$ & $34.7 \pm 6.9$ & $\begin{array}{l}27.2 \pm 6.0 \mathrm{mo} \\
\quad \text { (range } 12-44 \\
\text { mo) }\end{array}$ & High (13/16) & $\begin{array}{l}\text { To evaluate patients' ability to } \\
\text { return to HIIT after hip } \\
\text { arthroscopic surgery for } \\
\text { FAIS }\end{array}$ \\
\hline $\begin{array}{l}\text { Matsuda et al., } \\
2014^{33}\end{array}$ & IV & Arthroscopy & FAI patients & 30 & 30 & $16 / 14$ & 37.8 & Intraoperative & Fair (10/16) & $\begin{array}{l}\text { To evaluate the concept of cam } \\
\text { FAI occurring medial to the } \\
\text { classic AL quadrant. } \\
\text { Hypothesis was that the } \\
\text { addition of anteromedial } \\
\text { femoroplasty would } \\
\text { improve hip internal } \\
\text { rotation beyond that } \\
\text { achieved with classical } \\
\text { anterolateral femoroplasty. }\end{array}$ \\
\hline $\begin{array}{l}\text { Choi et al., } \\
2018^{30}\end{array}$ & IV & $\begin{array}{l}\text { Journal of the } \\
\text { American } \\
\text { Academy of } \\
\text { Orthopaedic } \\
\text { Surgeons }\end{array}$ & $\begin{array}{l}\text { FAI patients (Asian } \\
\text { population) }\end{array}$ & 109 & 109 & $39 / 70$ & 44.2 & $\begin{array}{l}27 \text { mo (range } 24- \\
54 \text { ) }\end{array}$ & Fair $(10 / 16)$ & $\begin{array}{l}\text { To evaluate an Asian cohort for } \\
\text { changes in ROM and clinical } \\
\text { function scales after they } \\
\text { underwent arthroscopic } \\
\text { femoroplasty of the hip }\end{array}$ \\
\hline $\begin{array}{l}\text { Kelly et al., } \\
2012^{32}\end{array}$ & III & $A J S M$ & $\begin{array}{l}\text { FAI patients } \\
\text { (specifically cam } \\
\text { presence) }\end{array}$ & 55 & 56 & $11 / 44$ & $24.7 \pm 6.3$ & $\begin{array}{l}\text { After } \\
\text { decompression } \\
\text { (day of surgery } \\
\text { in operating } \\
\text { room) and again } \\
\text { 3-mo } \\
\text { postoperative }\end{array}$ & Fair (10/16) & $\begin{array}{l}\text { To determine the alteration in } \\
\text { rotation of the hip after } \\
\text { arthroscopic cam } \\
\text { decompression, as assessed } \\
\text { by correction of the alpha } \\
\text { angle. To describe the role of } \\
\text { femoral neck version in } \\
\text { determining hip rotation in } \\
\text { the setting of FAI and } \\
\text { arthroscopic cam } \\
\text { decompression and to } \\
\text { determine whether } \\
\text { improvement in internal } \\
\text { rotation can be achieved } \\
\text { independent of the } \\
\text { underlying femoral version }\end{array}$ \\
\hline
\end{tabular}




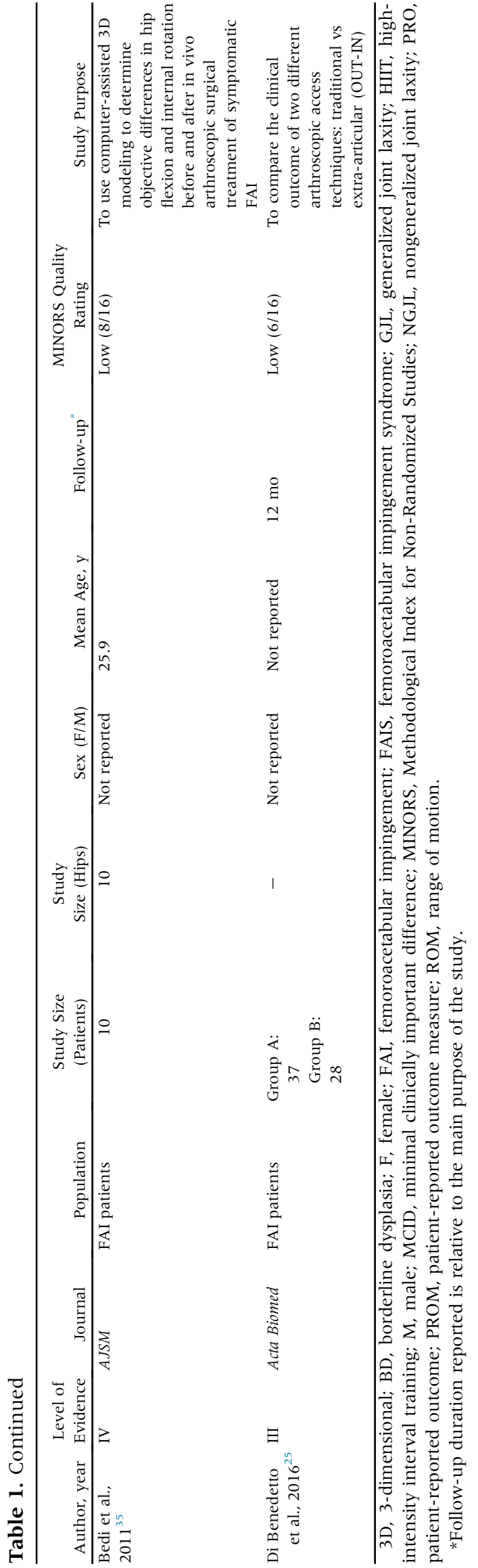

methodologic quality scores ranged from 4 to 13 of 16 for noncomparative studies and from 17 to 19 points of 24 for comparative studies (Appendix Table 2, available at www.arthroscopyjournal.org). The Kappa inter-rater agreement value was 0.822 (95\% CI 0.587-1.057), indicating excellent agreement between the 2 reviewers (D.F. and K.M.).

\section{Technique of ROM Evaluation}

In total, $52 \%$ of the included studies did not describe the technique with which any of the ROMs were assessed. Eight of $23(35 \%)$ of the included studies described the use of a goniometer as the primary ROM measurement technique, with 5 of these studies specifying the use of a manual/handheld goniometer. Of those that used goniometric evaluation, 2 studies $^{27,28}$ reported dual-operator evaluation, 1 study ${ }^{29}$ evaluated with a single operator, and the remaining $5^{30-34}$ did not distinguish. In 1 additional study, ${ }^{35}$ although not the primary purpose of the study, the authors did also report single-operator goniometric evaluation of ROM at 3-month clinical follow-up. Certainty of evidence for studies that used goniometric assessment of hip ROM was very low (Appendix Table 3, available at www.arthroscopyjournal.org).

In total, 3 of $23(13 \%)$ of the included studies ${ }^{35-37}$ assessed ROM via computed tomography (CT) simulation using a 3-dimensional-generated model. During simulated ROM maneuvers, the pelvis was fixed in space while the femur was free to move in a specified motion of interest. The resultant point of osseous collision between the proximal femur and acetabulum represented the range of motion in degrees. Certainty of evidence for studies using CT-simulated assessment of hip ROM was low (Appendix Table 4, available at www.arthroscopyjournal.org).

\section{Hip ROM}

ROM measures reported most frequently were flexion (21/23), internal rotation (IR) (23/23), and external rotation (ER) (17/25). Less frequently reported was abduction (4/23), adduction (3/23), total ROM ( $1 /$ $23)$, and extension (1/23).

The combination of flexion, IR, and ER was exclusively reported in 10 studies $^{29-31,34,38-43}$ and in conjunction with additional measurements in 5 further studies. ${ }^{25,27,28,32,44}$ Five studies reported flexion and IR only, ${ }^{37,45-47}$ with 1 further study assessing these measurements in addition to IR at $90^{\circ}$ hip flexion with $15^{\circ}$ adduction [FADIR]. ${ }^{36}$ The remaining 2 studies reported IR only. 33,48

The measured pre- and postoperative ROMs and corresponding estimates of effect size (SMD) are presented in Table 2. Heterogeneity of the measurement techniques used, patient demographics, follow-up duration, methodologic quality, and low certainty of 


\begin{tabular}{|c|c|c|c|c|c|c|c|}
\hline Author (year) & Study Size & ROM Assessed & Preoperative & Postoperative & $P$ Value & SMD $(95 \%$ CI $)[\text { Size }]^{\dagger}$ & $\begin{array}{l}\text { Technique Used to } \\
\text { Measure ROM }\end{array}$ \\
\hline Keating et al., & 22 patients & Flexion & $114.4 \pm 8.4$ & $120.5 \pm 6.9$ & Flexion $(P=.004)$ & $0.78(0.16-1.39)$ [moderate] & Not described \\
\hline \multirow[t]{2}{*}{$2020^{39}$} & & External rotation & $39.6 \pm 7.9$ & $40.6 \pm 4.6$ & $\begin{array}{l}\text { External rotation } \\
\quad(P=.50, \mathrm{NS})\end{array}$ & $0.15(-0.44$ to 0.74$)$ & \\
\hline & & Internal rotation & $18.0 \pm 6.8$ & $24.6 \pm 6.8$ & Internal rotation $(P=.001)$ & $0.95(0.33-1.58)$ [large] & \\
\hline \multirow{2}{*}{$\begin{array}{l}\text { Ragab et al., } \\
2018^{46}\end{array}$} & 40 patients & Flexion & $92.88 \pm 4.79$ & $105.63 \pm 8.26$ & Flexion $(P<.001)$ & $1.86(1.33-2.38)$ [large] & Internal rotation at $90^{\circ}$ hip \\
\hline & & Internal rotation & $8.25 \pm 7.30$ & $14.74 \pm 6.40$ & $\begin{array}{l}\text { Internal rotation } \\
\quad(P<.001)\end{array}$ & $0.92(0.46-1.39)$ [large] & $\begin{array}{c}\text { flexion, but measurement } \\
\text { instrument/technique not } \\
\text { described. }\end{array}$ \\
\hline \multirow{3}{*}{$\begin{array}{l}\text { Waterman } \\
\text { et al., } \\
2018^{43}\end{array}$} & 29 patients & Flexion & $110.3 \pm 11.4$ & $117.1 \pm 8.4$ & Flexion $(P=.01)$ & $0.67(0.14-1.20)$ [moderate] & Not described \\
\hline & & External rotation & $39.2 \pm 8.5$ & $40.5 \pm 11.1$ & $\begin{array}{l}\text { External rotation } \\
\quad(P=.608, \mathrm{NS})\end{array}$ & $0.13(-0.37$ to 0.63$)$ & \\
\hline & & Internal rotation & $12.6 \pm 9.9$ & $21.0 \pm 9.6$ & $\begin{array}{l}\text { Internal rotation }(P= \\
.0001)\end{array}$ & $0.85(0.31-1.39)$ [large] & \\
\hline \multirow{10}{*}{$\begin{array}{l}\text { Flores et al., } \\
2018^{44}\end{array}$} & 58 patients & & Early group: & Early group: & Early group: & & Not described \\
\hline & 60 hips & Flexion & $115.9 \pm 6.3$ & $118.4 \pm 4.8$ & Flexion $(P=.085, \mathrm{NS})$ & $0.44(-0.07$ to 0.95$)$ & \\
\hline & & Extension & $8.4 \pm 3.6$ & $9.3 \pm 2.6$ & Extension $(P=.296$, NS $)$ & $0.28(-0.23$ to 0.79$)$ & \\
\hline & & Internal rotation & $15.2 \pm 8.2$ & $27.1 \pm 5.4$ & Internal rotation $(P<.0001)$ & $1.69(1.10-2.29)$ [large] & \\
\hline & & External rotation & $49.8 \pm 7.1$ & $46.6 \pm 6.0$ & $\begin{array}{l}\text { External rotation } \\
\qquad(P=.064, \mathrm{NS})\end{array}$ & $-0.48(-0.99$ to 0.03$)$ & \\
\hline & & & Late group: & Late group: & Late group: & & \\
\hline & & Flexion & $113.4 \pm 11.2$ & $118.0 \pm 4.8$ & Flexion $(P=.052, \mathrm{NS})$ & $0.53(0.01-1.04)$ & \\
\hline & & Extension & $9.6 \pm 3.3$ & $9.8 \pm 0.9$ & Extension $(P=.786, \mathrm{NS})$ & $0.08(-0.42$ to 0.59$)$ & \\
\hline & & Internal rotation & $19.5 \pm 5.8$ & $28.0 \pm 3.7$ & $\begin{array}{l}\text { Internal rotation } \\
\quad(P<.0001)\end{array}$ & $1.72(1.13,2.32)$ [large] & \\
\hline & & External rotation & $45.0 \pm 6.7$ & $46.1 \pm 2.5$ & $\begin{array}{l}\text { External rotation } \\
\quad(P=.431, \mathrm{NS})\end{array}$ & $0.21(-0.29$ to 0.72$)$ & \\
\hline \multirow{6}{*}{$\begin{array}{l}\text { Carton and } \\
\text { Filan, } \\
2020^{27}\end{array}$} & 576 hips $(\mathrm{n}=410$ & Flexion & $111.0 \pm 11.2$ & $117.5 \pm 8.9$ & Flexion $(P<.001)$ & $0.74(0.60-0.88)$ [moderate] & Dual operator, hand-held \\
\hline & with ROM follow- & Abduction & $44.8 \pm 9.0$ & $48.8 \pm 8.7$ & Abduction $(P<.001)$ & $0.45(0.31-0.59)$ [weak] & goniometer \\
\hline & up) & Adduction & $20.3 \pm 7.8$ & $24.3 \pm 6.1$ & Adduction $(P<.001)$ & $0.57(0.43-0.71)$ [moderate] & \\
\hline & & External rotation & $37.6 \pm 8.3$ & $40.3 \pm 7.5$ & $\begin{array}{l}\text { External rotation } \\
\quad(P<.001)\end{array}$ & $0.34(0.20-0.48)[$ weak $]$ & \\
\hline & & Internal rotation & $23.5 \pm 10.9$ & $31.2 \pm 9.2$ & $\begin{array}{l}\text { Internal rotation } \\
\quad(P<.001)\end{array}$ & $0.76(0.62-0.90)$ [moderate] & \\
\hline & & Total ROM & $237.2 \pm 31.7$ & $262.1 \pm 27.8$ & Total ROM $(P<.001)$ & $0.83(0.69-0.98)$ [large] & \\
\hline \multirow{8}{*}{$\begin{array}{l}\text { Stone et al., } \\
2019^{42}\end{array}$} & 125 patients $(25$ & & GJL group: & GJL group: & GJL group: & $0.60(0.03-1.17)$ [moderate] & Single operator (senior \\
\hline & GJL and 100 no & Flexion & $118 \pm 10.7$ & $124 \pm 8.93$ & Flexion: $(P=.025)$ & & author), goniometer, \\
\hline & GJL) & External rotation & $50.0 \pm 11.1$ & $48.1 \pm 13.3$ & External rotation: (NS) & $-0.15(-0.71,0.40)$ & external rotation and \\
\hline & & Internal rotation & $17.9 \pm 9.8$ & $25.5 \pm 5.17$ & $\begin{array}{l}\text { Internal rotation: } \\
\quad(P<.001)\end{array}$ & $0.95(0.37-1.54)$ [large] & $\begin{array}{l}\text { internal rotation with hip } \\
\text { flexed to } 90^{\circ}\end{array}$ \\
\hline & & & No-GJL group: & No-GJL group: & No-GJL group: & $0.65(0.37-0.94)$ [moderate] & \\
\hline & & Flexion & $113 \pm 13.6$ & $120 \pm 6.6$ & Flexion: $(P=.003)$; & & \\
\hline & & External rotation & $44.6 \pm 10.5$ & $45.2 \pm 11.9$ & External rotation: (NS) & $0.05(-0.22,0.33)$ & \\
\hline & & Internal rotation & $17.3 \pm 11.0$ & $23.0 \pm 6.1$ & $\begin{array}{l}\text { Internal rotation: } \\
\quad(P<.001)\end{array}$ & $0.64(0.35-0.92)$ [moderate] & \\
\hline
\end{tabular}




\begin{tabular}{|c|c|c|c|c|c|c|c|}
\hline Author (year) & Study Size & ROM Assessed & Preoperative & Postoperative & $P$ Value & SMD $(95 \%$ CI $)[\text { Size }]^{\dagger}$ & $\begin{array}{l}\text { Technique Used to } \\
\text { Measure ROM }\end{array}$ \\
\hline \multirow[t]{3}{*}{$\begin{array}{l}\text { Ross et al., } \\
2018^{38}\end{array}$} & \multirow[t]{3}{*}{$\begin{array}{l}13 \text { patients (17 } \\
\text { hips) }\end{array}$} & Flexion & $\begin{array}{c}108.2 \pm 15.3 \text { (range } 73- \\
127 \text { ) }\end{array}$ & $114.8 \pm 12.1$ (range 94-135) & Flexion $(P<.001)$ & $0.47(-0.22,1.15)[$ weak $]$ & \multirow{3}{*}{$\begin{array}{l}\text { CT-simulated ROM using } \\
\text { 3D-generated model } \\
\text { (pelvis fixed in space, } \\
\text { femur rotated until contact } \\
\text { between the femur and the } \\
\text { acetabulum occurred, } \\
\text { causing a femoral head } \\
\text { translation). Specifically, } \\
\text { measured direct hip } \\
\text { flexion; internal rotation in } \\
90^{\circ} \text { flexion; internal } \\
\text { rotation in } 90^{\circ} \text { hip flexion } \\
\text { with } 15^{\circ} \text { adduction. }\end{array}$} \\
\hline & & Internal rotation & $\begin{array}{l}20.5 \pm 17.4 \\
\text { (range } 0-52 \text { ) }\end{array}$ & $31.8 \pm 16.4($ range $7-58)$ & $\begin{array}{l}\text { Internal rotation } \\
\quad(P<.001)\end{array}$ & $\begin{array}{l}0.65(-0.04,1.34) \\
\quad[\text { moderate }]\end{array}$ & \\
\hline & & IR + adduction & $12.3 \pm 13.3($ range $0-39)$ & $22.9 \pm 16.2($ range $0-47)$ & $\begin{array}{l}\mathrm{IR}+\text { adduction } \\
\quad(P<.001)\end{array}$ & 0.70 (0.00-1.39) [moderate] & \\
\hline $\begin{array}{l}\text { Polesello et al., } \\
2009^{48}\end{array}$ & 28 patients & Internal rotation & $\begin{array}{c}17 \pm 16.9 \text { (range }-15 \text { to } \\
45)\end{array}$ & $\begin{array}{c}36 \pm 11.6(\text { range } 0-50) \\
\Delta \text { Internal rotation }=19 \\
\quad(\text { range } 0-40)\end{array}$ & $\begin{array}{l}\text { Internal rotation } \\
\quad(P<.001)\end{array}$ & $1.29(0.71-1.87)$ [large] & $\begin{array}{l}\text { Supine position with } 90^{\circ} \\
\text { flexion and maximum } \\
\text { internal rotation }\end{array}$ \\
\hline \multirow{5}{*}{$\begin{array}{l}\text { Mullins et al., } \\
2020^{28}\end{array}$} & 47 patients (70 & Flexion & $116.7 \pm 8.7$ & $117.2 \pm 6.9$ & flexion (NS) & $0.06(-0.40$ to 0.53$)$ & \multirow{5}{*}{$\begin{array}{l}\text { Dual-operator, hand-held } \\
\text { goniometer }\end{array}$} \\
\hline & hips) - 36 athletes & Abduction & $50.9 \pm 9.8$ & $52.2 \pm 6.4$ & Abduction (NS) & $0.16(-0.31$ to 0.62$)$ & \\
\hline & returned for 1-year & Adduction & $24.6 \pm 6.1$ & $27.8 \pm 2.8$ & $\begin{array}{l}\text { Adduction } \\
\qquad(P=.012)\end{array}$ & $0.67(0.19-1.14)$ [moderate] & \\
\hline & & External rotation & $38.7 \pm 7.6$ & $44.5 \pm 5.3$ & $\begin{array}{l}\text { External rotation } \\
\quad(P<.001)\end{array}$ & $0.88(0.39-1.36)$ [large] & \\
\hline & & Internal rotation & $23.8 \pm 8.5$ & $27.4 \pm 3.9$ & Internal rotation $(P=.003)$ & $0.54(0.07-1.01)$ [moderate] & \\
\hline $\begin{array}{l}\text { Stone et al., } \\
2019^{42}\end{array}$ & 688 patients & $\begin{array}{l}\text { Flexion } \\
\text { External rotation } \\
\text { Internal rotation }\end{array}$ & $\begin{array}{l}\text { Quantitative values not } \\
\text { reported-bar graph with } \\
\text { values displayed }\end{array}$ & $\begin{array}{l}\text { Quantitative values not } \\
\text { reported-bar graph with } \\
\text { values displayed }\end{array}$ & $\begin{array}{l}\text { Not reported (preoperative to } \\
\text { postoperative not reported, } \\
\text { only the significance of } \\
\text { difference between groups } \\
\text { at postoperative time point) }\end{array}$ & $\begin{array}{l}- \\
- \\
-\end{array}$ & Not described \\
\hline \multirow{3}{*}{$\begin{array}{l}\text { Frank et al., } \\
2018^{38} \text { (yoga) }\end{array}$} & 42 patients & Flexion & $111.81 \pm 10.83$ & $119.23 \pm 8.15$ & Flexion $(P=.0025)$ & $0.77(0.34-1.19)$ [moderate] & \multirow[t]{3}{*}{ Not described } \\
\hline & 45 hips & Internal rotation & $19.17 \pm 7.32$ & $23.46 \pm 5.64$ & Internal rotation $(P=.001)$ & $0.66(0.23-1.08)$ [moderate] & \\
\hline & & External rotation & $39.2 \pm 8.5$ & $40.5 \pm 11.1$ & $\begin{array}{l}\text { External rotation } \\
\qquad(P=.608, \mathrm{NS})\end{array}$ & $0.13(-0.28$ to 0.54$)$ & \\
\hline \multirow{2}{*}{$\begin{array}{l}\text { Frank et al., } \\
2018^{45} \\
\text { (cyclists) }\end{array}$} & 58 patients & Flexion & $110.3 \pm 11.4$ & $118.1 \pm 8.44$ & Flexion $(P<.01)$ & $0.77(0.40-1.15)$ [moderate] & \multirow[t]{2}{*}{ Not described } \\
\hline & 60 hips & Internal rotation & $12.58 \pm 9.91$ & $20.97 \pm 9.62$ & Internal rotation $(P<.001)$ & $0.86(0.48-1.23)[$ large] & \\
\hline Levy et al., & 84 patients $(28$ & & Atypical group: & Atypical group: & Not reported. Statistical & & Not described \\
\hline \multirow{6}{*}{$2017^{40}$} & atypical; 56 typical) & Flexion & $110 \pm 20.9$ & $126 \pm 12.3$ & significance between typical & $0.86(0.31-1.41)$ & \\
\hline & & External rotation & $44.1 \pm 12.1$ & $47.1 \pm 6.6$ & and atypical groups only & $0.30(-0.22$ to 0.83$)$ & \\
\hline & & Internal rotation & $\begin{array}{c}16.6 \pm 11.9 \\
\text { Typical group: }\end{array}$ & $\begin{array}{c}21.1 \pm 8.9 \\
\text { Typical group: }\end{array}$ & $\begin{array}{l}\text { reported, not the change from } \\
\text { baseline }\end{array}$ & $0.42(-0.11$ to 0.95$)$ & \\
\hline & & Flexion & $114 \pm 13.2$ & $118 \pm 14.9$ & & $0.28(-0.09$ to 0.65$)$ & \\
\hline & & External rotation & $43.0 \pm 9.2$ & $43.1 \pm 10.4$ & & $0.01(-0.36$ to 0.38$)$ & \\
\hline & & Internal rotation & $14.6 \pm 11.9$ & $22.0 \pm 5.5$ & & $0.79(0.41-1.18)$ & \\
\hline
\end{tabular}




\begin{tabular}{|c|c|c|c|c|c|c|c|}
\hline Author (year) & Study Size & ROM Assessed & Preoperative & Postoperative & $P$ Value ${ }^{*}$ & $\operatorname{SMD}(95 \%$ CI $)[\text { Size }]^{\dagger}$ & $\begin{array}{l}\text { Technique Used to } \\
\text { Measure ROM }\end{array}$ \\
\hline \multirow{8}{*}{$\begin{array}{l}\text { Nawabi et al., } \\
2016^{41}\end{array}$} & BD group, 46 cases & & BD group: & BD group: & Not reported. Statistical & & Not described \\
\hline & Control. & Flexion & $108.1 \pm 7.3$ & $105.9 .1 \pm 5.3$ & significance between BD group & $-0.34(-0.75$ to 0.07$)$ & \\
\hline & 131 cases & External rotation & $41.9 \pm 5.8$ & $42.2 \pm 3.6$ & and control group only & $0.06(-0.35$ to 0.47$)$ & \\
\hline & & Internal rotation & $14 \pm 11.6$ & $25.4 \pm 4.9$ & reported at preoperative and & $1.27(0.82-1.72)$ & \\
\hline & & & Control group: & Control group: & postoperative separately & & \\
\hline & & Flexion & $107 \pm 9.9$ & $104.5 \pm 5.7$ & & $-0.31(-0.55$ to -0.06$)$ & \\
\hline & & External rotation & $43 \pm 10.2$ & $44.2 \pm 6.9$ & & $0.14(-0.11$ to 0.38$)$ & \\
\hline & & Internal rotation & $13.5 \pm 11.9$ & $27.5 \pm 4.6$ & & $1.55(1.27-1.82)$ & \\
\hline \multirow{14}{*}{$\begin{array}{l}\text { Fabricant et al., } \\
2015^{31}\end{array}$} & 243 cases $(243$ & & Decreased version: & No postoperative values & Not reported as a statistically & & Flexion, internal, and \\
\hline & patients) & Internal rotation & $6 \pm 6$ & reported, only mean change & significant change from & $3.33(2.26-4.41)$ & external rotation at $90^{\circ}$ of \\
\hline & ROM available for & Flexion & $104 \pm 7$ & from baseline & preoperative to postoperative & $1.0(0.0-0.0)$ & flexion was measured \\
\hline & 227 cases & External rotation & $\begin{array}{l}44 \pm 10 \\
\text { Normal version: }\end{array}$ & $\begin{array}{c}\text { Decreased version: } \\
\Delta \text { internal rotation: } 20 \pm 7\end{array}$ & $\begin{array}{l}\text { scores within the different } \\
\text { groups. Only reports }\end{array}$ & $-0.20(-0.14$ to 0.26$)$ & using a goniometer \\
\hline & & Internal rotation & $12 \pm 8$ & $\Delta$ flexion: $0 \pm 8$ & significance between the 3 & $1.88(1.57-2.18)$ & \\
\hline & & Flexion & $105 \pm 8$ & $\Delta$ external rotation: $-2 \pm 12$ & groups at either of the time & -0.13 ( -0.1 to 0.15 & \\
\hline & & External rotation & $42 \pm 9$ & Normal version: & points. & $0.22(0.19-0.26)$ & \\
\hline & & & Increased version & $\Delta$ internal rotation: $15 \pm 8$ & & & \\
\hline & & Internal rotation & $22 \pm 15$ & $\Delta$ flexion: $-1 \pm 8$ & & $0.67(0.49-0.84)$ & \\
\hline & & Flexion & $109 \pm 8$ & $\Delta$ external rotation: $2 \pm 9$ & & $-0.63(-0.46$ to 0.79 & \\
\hline & & External rotation & $42 \pm 10$ & Increased version: & & $0.30(0.22-0.38)$ & \\
\hline & & & & $\Delta$ internal rotation: $10 \pm 15$ & & & \\
\hline & & & & $\Delta$ flexion: $-5 \pm 17$ & & & \\
\hline & & & & $\Delta$ external rotation: $3 \pm 11$ & & & \\
\hline \multirow[t]{6}{*}{$\begin{array}{l}\text { Ross et al., } \\
2015^{37}\end{array}$} & $\begin{array}{l}\text { Revision group: } \\
47 \text { patients }(50\end{array}$ & & $\begin{array}{l}\text { Revision group (prior to } \\
\text { revision): }\end{array}$ & $\begin{array}{l}\text { Revision group (after virtual } \\
\text { revision } \mathrm{Sx} \text { ) }\end{array}$ & Revision group & & $\begin{array}{l}\text { CT-simulated ROM using a } \\
\text { 3D-generated model }\end{array}$ \\
\hline & hips) & Flexion & $114 \pm 14($ range $78-145)$ & $121 \pm 11($ range $97-145)$ & Flexion $(P<.001$ & $0.55(0.15-0.95)$ [moderate] & During the simulated ROM \\
\hline & $\begin{array}{l}\text { Nonrevision group: } \\
65 \text { patients }(65\end{array}$ & Internal rotation & $28 \pm 15$ (range $0-60)$ & $34 \pm 13($ range $8-60)$ & $\begin{array}{c}\text { Internal rotation } \\
(P<.001)\end{array}$ & $0.42(0.03-0.82)[$ weak] & $\begin{array}{l}\text { maneuvers, the femur was } \\
\text { moved in a specific motion }\end{array}$ \\
\hline & hips) & & Nonrevision group: & $\begin{array}{l}\text { Nonrevision group (CT- } \\
\text { simulated measured ROM for } \\
\text { actual postoperative: }\end{array}$ & Nonrevision group: & & $\begin{array}{l}\text { until contact between the } \\
\text { femur and acetabulum } \\
\text { occurred (detected by the }\end{array}$ \\
\hline & & Flexion & $121 \pm 11$ & $129 \pm 10($ range $105-155)$ & Flexion $(P<.001)$ & $0.76(0.40-1.11)$ [moderate] & resultant translation of the \\
\hline & & Internal rotation & $35 \pm 13$ & $49 \pm 11($ range $25-90)$ & $\begin{array}{l}\text { Internal rotation } \\
\quad(P<.001)\end{array}$ & $1.16(0.78-1.53)$ [large] & $\begin{array}{l}\text { femoral head). The point of } \\
\text { osseous collision was } \\
\text { defined as the occurrence } \\
\text { of mechanical } \\
\text { impingement, } \\
\text { which was recorded in } \\
\text { degrees of motion. }\end{array}$ \\
\hline \multirow{5}{*}{$\begin{array}{l}\text { Stähelin et al., } \\
2008^{47 \ddagger} \\
\text { Riff et al., } \\
2018^{34}\end{array}$} & 14 patients & Flexion & $112 \pm 14.1$ & $132 \pm 8.0$ & Not reported & $1.69(0.81-2.58)$ [large] & Supine position \\
\hline & & Internal rotation & $8 \pm 8.0$ & $19 \pm 11.0$ & & $1.11(0.31-1.91)$ [large] & \\
\hline & 32 patients & Flexion & $111.4 \pm 10.0$ & $120.8 \pm 5.6$ & Flexion $(P<.001)$ & $1.15(0.65-1.64)$ [large] & $90^{\circ}$ of hip flexion with a \\
\hline & 37 hips & External rotation & $39.7 \pm 11.5$ & $41.4 \pm 8.4$ & $\begin{array}{l}\text { External rotation } \\
\quad(P=.50, \mathrm{NS})\end{array}$ & $0.17(-0.29$ to 0.62$)$ & handheld goniometer \\
\hline & & Internal rotation & $11.1 \pm 8.8$ & $21.7 \pm 7.5$ & $\begin{array}{l}\text { Internal rotation } \\
\quad(P<.001)\end{array}$ & $1.28(0.78-1.79)[$ large $]$ & \\
\hline
\end{tabular}




\begin{tabular}{|c|c|c|c|c|c|c|c|}
\hline Author (year) & Study Size & ROM Assessed & Preoperative & Postoperative & $P$ Value ${ }^{*}$ & SMD $(95 \%$ CI $)[\text { Size }]^{\dagger}$ & $\begin{array}{l}\text { Technique Used to } \\
\text { Measure ROM }\end{array}$ \\
\hline $\begin{array}{l}\text { Matsuda et al., } \\
2014^{33}\end{array}$ & 30 patients & Internal rotation & $20.8(10-29)$ & $\begin{array}{c}\text { After AL femoroplasty } \\
29.5(18-39)[\Delta 8.7] \\
\text { After AM femoroplasty: } \\
42.7(32-61)[\Delta 13.2] \\
\text { After AL+AM femoroplasty: } \\
42.7(32-61)[\Delta 21.9]\end{array}$ & $\begin{array}{l}\text { After AL femoroplasty } \\
(P<.0001) \\
\text { After AM femoroplasty } \\
(P<.0001) \\
\text { After AL }+ \text { AM } \\
\text { femoroplasty } \\
(P<.0001)\end{array}$ & $\begin{array}{l}5.30(4.20-6.40)[\text { large] } \\
5.30(4.20-6.40)[\text { large }]\end{array}$ & $\begin{array}{c}\text { Intraoperative dynamic } \\
\text { testing with hip at } 90^{\circ} \\
\text { flexion and } 0^{\circ} \text { adduction } \\
\text { using large metal } \\
\text { goniometer placed in the } \\
\text { center of the patella with } \\
\text { one arm on the pretibial } \\
\text { crest and the other aligned } \\
\text { with the longitudinal axis } \\
\text { of the patient. A surgical } \\
\text { assistant performed } \\
\text { internal rotation }\end{array}$ \\
\hline $\begin{array}{l}\text { Choi et al., } \\
2018^{30}\end{array}$ & $\begin{array}{l}109 \text { patients } \\
109 \text { hips }\end{array}$ & $\begin{array}{l}\text { Flexion } \\
\text { Internal rotation } \\
\text { External rotation }\end{array}$ & $\begin{array}{c}106.3 \pm 9.3 \\
13.5 \pm 6.9 \\
22.6 \pm 8.5\end{array}$ & $\begin{array}{c}\text { Flexion: } \\
3 \text { mo: } 106.6 \pm 9.4 \\
6 \text { mo: } 108.1 \pm 9.2 \\
1 \text { y: } 108.4 \pm 9.0 \\
2 \text { y: } 106.4 \pm 4.0 \\
\text { Internal rotation: } \\
3 \text { mo: } 23.6 \pm 7.0 \\
6 \text { mo: } 25.2 \pm \pm 7.4 \\
1 \text { y: } 25.2 \pm 7.1 \\
2 \text { y: } 24.9 \pm 8.0 \\
\text { External rotation: } \\
3 \text { mo: } 23.1 \pm 8.0 \\
6 \text { mo: } 34.4 \pm 7.4 \\
1 \text { y: } 34.6 \pm 7.2 \\
2 \text { y: } 35.4 \pm 5.2\end{array}$ & $\begin{array}{c}\text { Between preoperative and } 2 \mathrm{y} \text { : } \\
\text { Flexion }(P=.92, \mathrm{NS}) \\
\text { Internal rotation }(\boldsymbol{P}=\mathbf{. 0 2}) \\
\text { External rotation }(\boldsymbol{P}=\mathbf{. 0 1})\end{array}$ & $\begin{array}{l}0.01(-0.25,0.28) \\
1.52(1.22-1.82)[\text { large] } \\
1.81(1.49-2.13) \text { [large] }\end{array}$ & $\begin{array}{c}\text { Internal rotation and } \\
\text { external rotation measured } \\
\text { at } 90^{\circ} \text { hip flexion, using a } \\
\text { manual goniometer }\end{array}$ \\
\hline $\begin{array}{l}\text { Kelly et al., } \\
2012^{32}\end{array}$ & $\begin{array}{c}55 \text { patients } \\
56 \text { hips }\end{array}$ & $\begin{array}{l}\text { Internal rotation } \\
\text { Internal rotation } \\
\text { Internal rotation } \\
\text { Internal rotation } \\
\text { External rotation } \\
\text { Flexion } \\
\text { Abduction } \\
\text { Internal rotation } \\
\text { Flexion } \\
\text { External rotation } \\
\text { Abduction }\end{array}$ & $\begin{array}{c}\text { (all): } 9.9 \pm 6.6 \\
\text { (increased FV): } 15.7 \pm 5.4 . \\
\text { (normal FV): } 10.6 \pm 5.4 . \\
\text { (decreased FV): } 7.1 \pm 8.3 \\
45.9 \pm 10.2 \\
115.7 \pm 13.3 \\
37.6 \pm 8.3\end{array}$ & $\begin{array}{c}\text { After decompression (day of } \\
\text { Sx) } \\
\text { (all): } 27.6 \pm 6.4 \\
\text { (increased FV): } 34.3 \pm 6.7 \\
\text { (normal FV): } 27.5 \pm 6.4 \\
\text { (decreased FV): } 25.2 \pm 4.9 \\
43.3 \pm 9.1 \\
108.8 \pm 9.8 \\
39.8 \pm 6.5 \\
3 \text { mo postoperative (all): } 30.1 \\
\pm 5.3 \\
127.9 \pm 6.6 \\
\text { Value not reported } \\
\text { Value not reported }\end{array}$ & 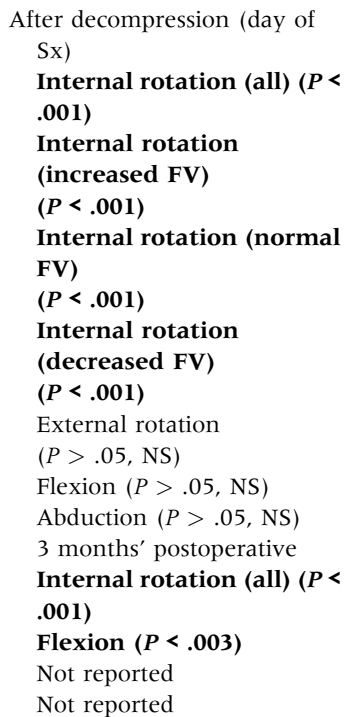 & $\begin{array}{l}2.70(2.19-3.22)[\text { large] } \\
2.89(1.38-4.40)[\text { large] } \\
2.82(2.14-3.50)[\text { large] } \\
2.57(1.49-3.65)[\text { large] } \\
-0.27(-0.64 \text { to } 0.10) \\
-0.59(-0.97 \text { to }-0.21) \\
0.29(-0.08 \text { to } 0.67) \\
3.35(2.77,3.93)[\text { large] } \\
1.15(0.75-1.56) \text { [large] } \\
- \\
-\end{array}$ & $\begin{array}{l}\text { Manual goniometer, } \\
\text { supine. Internal rotation } \\
\text { and external rotation } \\
\text { measured at } 90^{\circ} \text { hip } \\
\text { flexion. Internal rotation } \\
\text { was measured by } \\
\text { rotating the hip until } \\
\text { just before elevation of } \\
\text { the pelvis. External } \\
\text { rotation was determined } \\
\text { as the degree of rotation } \\
\text { with leg weight or } \\
\text { gravity only. }\end{array}$ \\
\hline
\end{tabular}




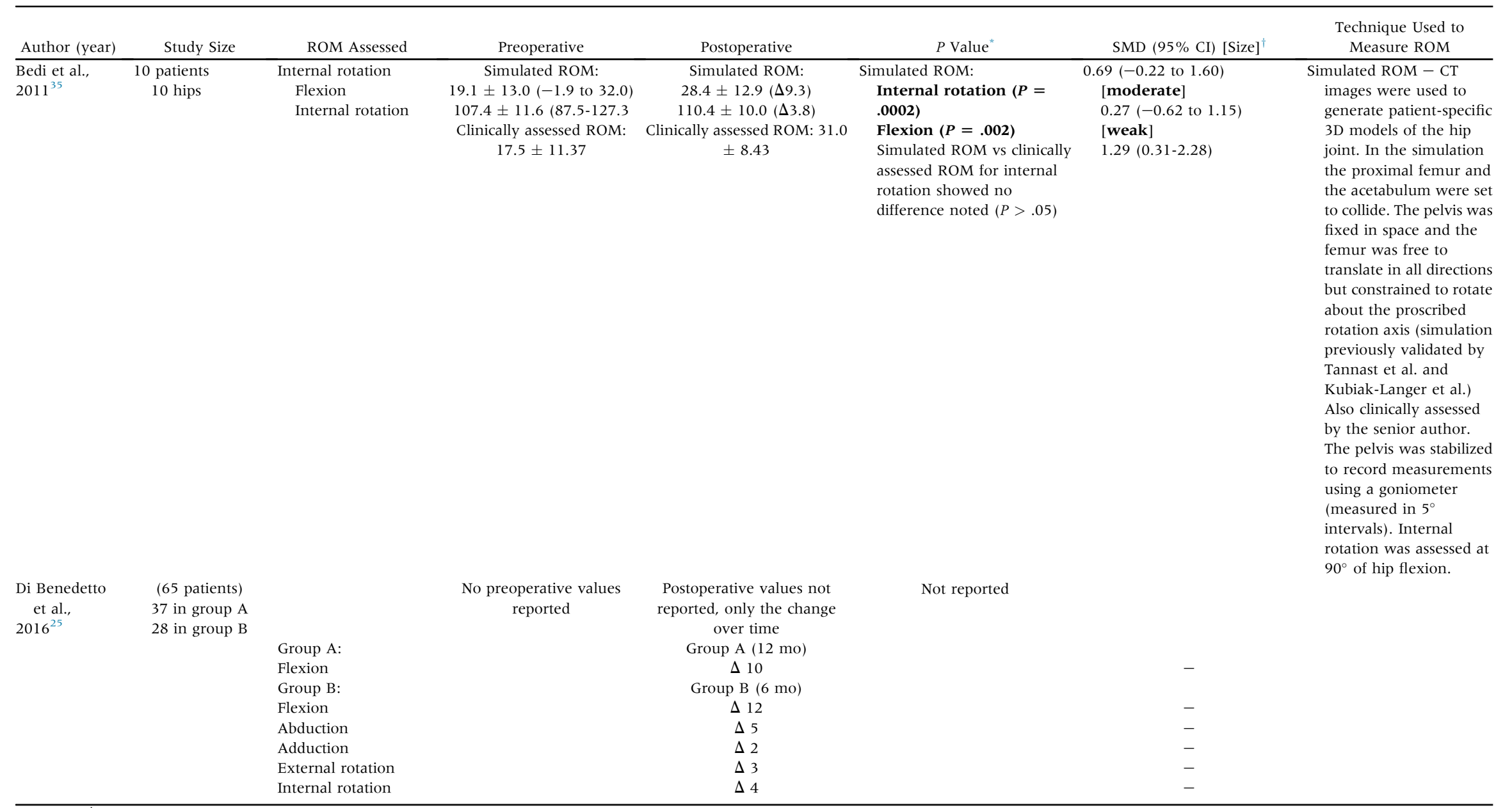

NOTE. $\Delta$ indicates the change in ROM from baseline to postoperative assessment. Significant changes from preoperative to postoperative are displayed in bold.

$\mathrm{AL}$, anterolateral; $\mathrm{AM}$, anteromedial; FV, femoral anteversion; ROM, range of motion; SMD, standardized mean difference.

${ }^{*} P$ value as reported in original study.

${ }^{\dagger}$ Size of SMD effect (weak, 0.2-0.49; moderate, 0.5-0.79; large, $>0.8$ ) is reported for those with statistically significant improvements preoperative to postoperative only.

${ }^{\ddagger}$ Stähelin et al. $2008^{47}$-this paper does contain Tönnis $2+$ but also reports specifically for those Tönnis $<2$, thus why it was included in this review. The $\mathrm{n}=14$ sample size reflects only those Tönnis 0 , where subanalysis of the entire cohort has been reported. 
evidence precluded meta-analysis to be undertaken in this review.

\section{Flexion}

Flexion was reported as an outcome measure in $91 \%$ $(\mathrm{n}=21)$ of the included studies. Postoperative changes in flexion from each study's baseline were reported as statistically significant in $57.1 \%(12 / 21)$, not statistically significant in $14.3 \%(3 / 21)$, and statistical significance was not reported in $28.6 \%(6 / 21)$. For those studies reporting statistically significant change in flexion, effect size was weak in $16.7 \%(2 / 12)^{35,36}$ of studies, moderate in $58.3 \%(7 / 123),{ }^{27,29,37-39,43,45}$ and large in $25 \%(3 / 12)^{32,34,46}$ of studies. Pre- to postoperative measured changes in flexion for studies evaluating unilaterally operated patients are presented in Figure $2 \mathrm{~A}$, whereas those studies evaluating a mix of unilateral and bilateral patients are presented in Figure 3A. Where simulated CT assessment was used, the postoperative measured change from baseline ranged from $3.0^{\circ}$ to $8.0^{\circ}$ for unilateral studies and $6.6^{\circ}$ in the study including bilateral patients. Where goniometric assessment was used, the postoperative measured change from baseline ranged from $0.1^{\circ}$ to $7.0^{\circ}$ (unilateral) and $5.0^{\circ}$ to $12.2^{\circ}$ (mixed). Where details of measurement technique were not provided, the postoperative measured change from baseline ranged from $6.1^{\circ}$ to $20.0^{\circ}$ (unilateral) and $-2.5^{\circ}$ to $7.8^{\circ}$ (mixed). Overall, for the majority, flexion trended to be higher postoperatively. In one study, ${ }^{41}$ the authors assessed ROM in 2 groups (borderline dysplasia vs no borderline dysplasia). For both these groups flexion was reduced postoperatively. Of note, while this study also had the longest period between comparative assessments (mean 31.3 months), it is unclear whether ROM clinical assessment was also undertaken at this time point or whether this postoperative period reflects the time point of PRO score, the major focus evaluation modality within this paper.

\section{Internal Rotation}

IR was reported as an outcome measure in $100 \%$ $(23 / 23)$ of studies. Postoperative changes in IR from each study's baseline were statistically significant in $74 \%(17 / 23)$, whereas statistical significance was not reported in $26 \%(6 / 23)$. For those studies reporting statistically significant improvements in IR, effect size was moderate in $29.4 \%(5 / 17)^{27,28,35,36,38}$ of studies, and large in $64.7 \%(11 / 17)^{30,32-34,37,39,43-46,48}$ of studies. One further study (1/17), ${ }^{29}$ evaluating 2 groups of patients, reported a moderate effect size in patients without generalized joint laxity and a large effect size in those with generalized joint laxity. Pre- to postoperative measured changes in IR are presented in Figure 2B, whereas those studies evaluating a mix of unilateral and bilateral patients are presented in Figure 3B. Where simulated CT assessment was used, the postoperative measured change from baseline ranged from $9.3^{\circ}$ to $14.0^{\circ}$ (unilateral) and $11.3^{\circ}$ (mixed). Where goniometric assessment was used, the postoperative measured change from baseline ranged from $5.7^{\circ}$ to $21.9^{\circ}$ (unilateral) and $3.6^{\circ}$ to $18.6^{\circ}$ (mixed). Where details of measurement technique were not provided, the postoperative measured change from baseline ranged from $6.5^{\circ}$ to $19.0^{\circ}$ (unilateral) and $4.3^{\circ}$ to $14.0^{\circ}$ (mixed). Across all studies, IR measured greater postoperatively. The study reporting the largest effect size assessed IR ROM intraoperatively. ${ }^{33}$

\section{External Rotation}

ER was reported in $65 \%(n=15)$ of the included studies. Postoperative changes in ER from each study's baseline were statistically significant in $20 \%(3 / 15)$, not statistically significant in $46.7 \%$ (7/15), and statistical significance was not reported in $33.3 \%$ (5/15). For those studies reporting statistically significant improvements in ER, effect size was weak in $33.3 \%$ (1/ $3)^{27}$ of studies and large in $66.7 \%(2 / 3),{ }^{28,30}$ of studies. Pre- to postoperative measured changes in external rotation are presented in Figure 2C, whereas those studies evaluating a mix of unilateral and bilateral patients are presented in Figure 3C. ER was not assessed in any of the CT simulation studies included. Where goniometric assessment was used, the postoperative measured change from baseline ranged from $0.6^{\circ}$ to $12.8^{\circ}$ (unilateral) and $-2.6^{\circ}$ to $5.8^{\circ}$ (mixed). In the study by Stone et al., ${ }^{29}$ ROM was reported for 2 groups (generalized joint laxity [GJL] vs no GJL). Although those without GJL had a mean increase on $0.6^{\circ}$ following surgery, those with GJL reported a reduced range for ER postoperatively $\left(-1.9^{\circ}\right)$. The largest gained increase in ER was reported by Choi et al. ${ }^{30}$ Of note, this study also reported the lowest pre- and postoperative mean value for this movement $\left(22.6^{\circ}\right.$ and $35.4^{\circ}$, respectively). Where details of measurement technique were not provided, the postoperative measured change from baseline was $1.0^{\circ}$ (unilateral) and ranged from $-3.2^{\circ}$ to $1.3^{\circ}$ (mixed). All but one of these studies reported increased measurements postoperatively, albeit to weak effect sizes. One study ${ }^{44}$ reported ROM for 2 groups (early vs later in surgeon's career). In this study ER was reduced $\left(-3.2^{\circ}\right)$ postoperatively in the early group, whereas there was a slight increase $\left(1.1^{\circ}\right)$ in the later operated group. While this measured change difference between groups was statistically significant, the change in ER from baseline to postoperative was not significantly different for either group.

\section{Quantification of Bony Deformity Correction}

In total, $13(57 \%)$ of the included studies reported pre- and postoperative alpha angle measurements, 


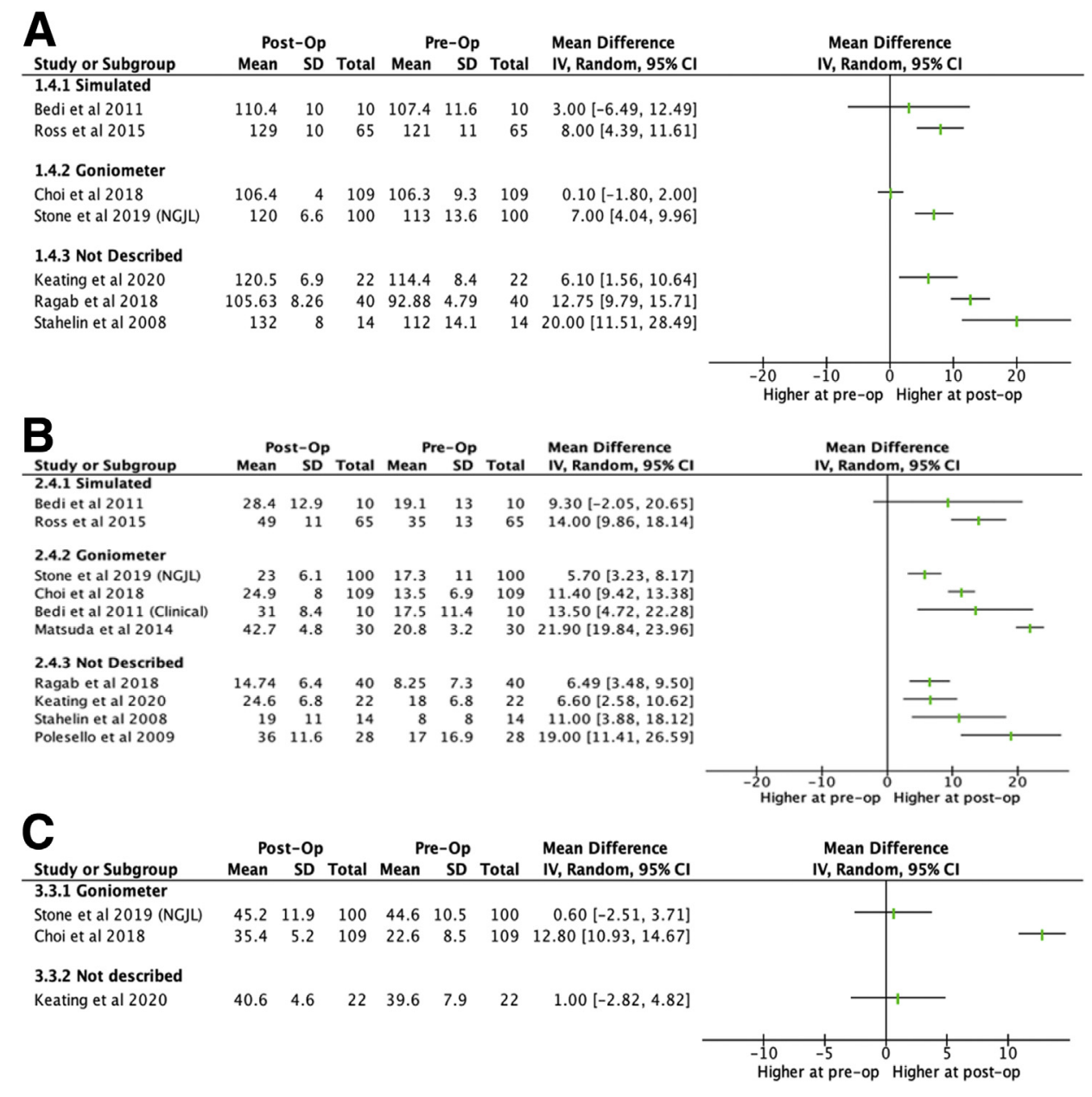

Fig 2. Forest plot of studies reporting ROM in unilaterally operated patients. The change in (A) flexion, (B) internal rotation, and $(\mathrm{C})$ external rotation following unilateral arthroscopic correction of femoroacetabular impingement (assessed using computed tomography simulation, goniometer and where exact technique not described) compared with preoperatively measured values. Overall heterogeneity (as assessed using $\mathrm{I}^{2}$ value) was $93 \%, 91 \%$ and $97 \%$ for flexion, internal rotation (IR) and external rotation (ER), respectively. (CI, confidence interval; IV, inverse variance; ROM, range of motion; SD, standard deviation.) quantifying the degree of femoroplasty relative to cam deformity correction ${ }^{27-30,32,34-39,44,45}$ (Table 3). Across the studies included, the mean extent of alpha angle correction (on any view) ranged from $1.7^{\circ}$ to $28.2^{\circ}$. Only 2 studies included in this review evaluated the impact of bony resection on ROM: Kelly et al. ${ }^{32}$ reported a change in alpha angle correlated with the magnitude of increase in IR $(r=0.35)$ whereas Stähelin et al. ${ }^{47}$ reported that neither postoperative alpha value or difference in alpha value achieved by correction correlated with any of the ascertained clinical parameters. Eight $(32 \%)$ of the included studies reported preand postoperative LCEA measurements, quantifying the degree of acetabuloplasty ${ }^{27-29,34,38,44,45}$ (Table 3). The mean extent of LCEA correction ranged from $1.2^{\circ}$ to $6.8^{\circ}$.

\section{Discussion}

All studies included in this review reported favorable ROM scores, with significant improvements from baseline postoperatively in at least one movement. Flexion, IR, and ER were the 3 most frequently reported measurements reported in $91 \%, 100 \%$, and
$65 \%$ of studies, respectively. Where the change in measurable ROM following hip arthroscopy was evaluated, the observed change was reported to be statistically significant in $57.1 \%$ (flexion), $74 \%$ (IR), and $20 \%$ (ER). In total, $52 \%$ of the included studies did not describe the technique with which ROM was evaluated. Where goniometric assessment was used, the mean change in predominant ROMs ranged as follows: flexion: $0.1^{\circ}$ to $12.2^{\circ}$; IR: $3.6^{\circ}$ to $21.9^{\circ}$; external rotation $-2.6^{\circ}$ to $12.8^{\circ}$. Where CT-simulated assessment was used, the mean change was as follows: flexion: $3.0^{\circ}$ to $8.0^{\circ}$; internal rotation: $9.3^{\circ}$ to $14.0^{\circ}$.

It is generally accepted that decreased motion in patients with symptomatic FAI occurs primarily because of a mechanical block to movement from abnormal bony morphology of the proximal femur and/or acetabulum. The extent of bony morphology can be quantified through radiographic analysis, measuring alpha angle and LCEA. Comparative and investigative studies for the majority have been focused on the cam morphology influencing ROM with studies demonstrating negative correlations between internal rotation and alpha angle. ${ }^{10,11,32,49}$ Further, the specific location 


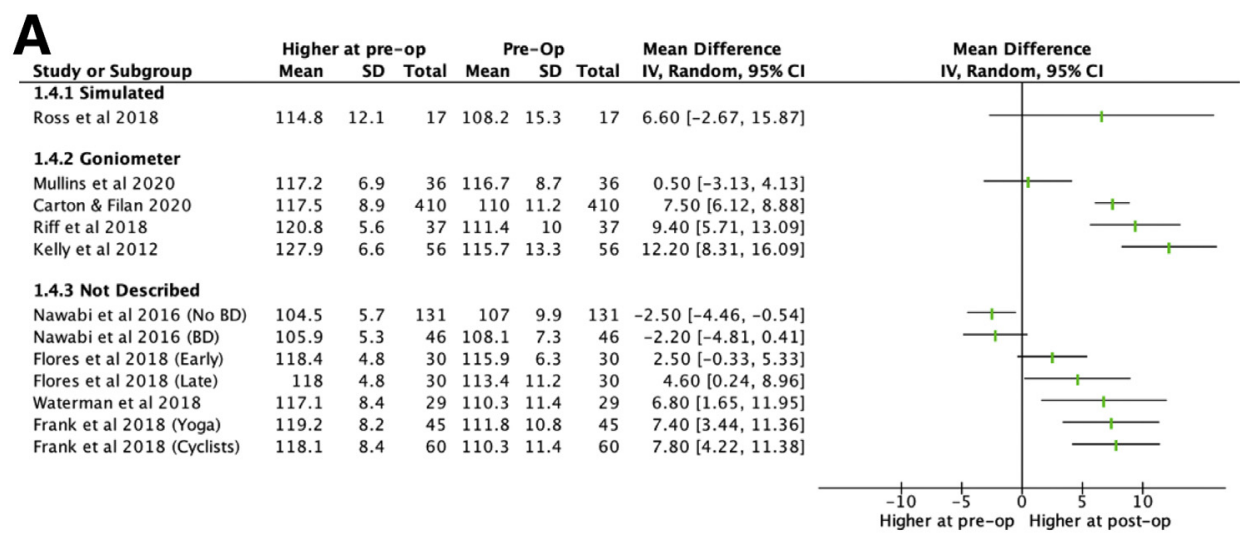

\section{B

\begin{tabular}{l} 
Study or Subgroup \\
\hline 2.4.1 Simulated \\
Ross et al 2018 \\
2.4.2 Goniometer \\
Mullins et al 2020 \\
Carton \& Filan 2020 \\
Riff et al 2018 \\
Kelly et al 2012 (Normal) \\
Kelly et al 2012 (Decreased) \\
Kelly et al 2012 (Increased) \\
2.4.3 Not Described \\
Frank et al 2018 (Yoga) \\
Frank et al 2018 (Cyclists) \\
Waterman et al 2018 \\
Flores et al 2018 (Late) \\
Nawabi et al 2016 (BD) \\
Flores et al 2018 (Early) \\
Nawabi et al 2016 (No BD)
\end{tabular}

\begin{tabular}{|c|c|c|c|c|c|c|}
\hline \multicolumn{3}{|c|}{ Post-Op } & \multicolumn{3}{|c|}{ Pre-Op } & \multirow{2}{*}{$\begin{array}{l}\text { Mean Difference } \\
\text { IV, Random, } 95 \% \mathrm{CI}\end{array}$} \\
\hline Mean & SD & Total & Mean & SD & Total & \\
\hline 31.8 & 16.4 & 17 & 20.5 & 17.4 & 17 & $11.30[-0.07,22.67]$ \\
\hline 27.4 & 3.9 & 36 & 23.8 & 8.5 & 36 & $3.60[0.55,6.65]$ \\
\hline 31.2 & 9.2 & 410 & 23.5 & 10.9 & 410 & $7.70[6.32,9.08]$ \\
\hline 21.7 & 7.5 & 37 & 11.1 & 8.8 & 37 & $10.60[6.87,14.33]$ \\
\hline 27.5 & 6.4 & 34 & 10.6 & 5.4 & 34 & $16.90[14.09,19.71]$ \\
\hline 25.2 & 4.9 & 13 & 7.1 & 8.3 & 13 & $18.10[12.86,23.34]$ \\
\hline 34.3 & 6.7 & 8 & 15.7 & 5.4 & 8 & $18.60[12.64,24.56]$ \\
\hline 23.5 & 5.6 & 45 & 19.2 & 7.3 & 45 & $4.30[1.61,6.99]$ \\
\hline 21 & 9.6 & 60 & 12.6 & 9.9 & 60 & $8.40[4.91,11.89]$ \\
\hline 21 & 9.6 & 29 & 12.6 & 9.9 & 29 & $8.40[3.38,13.42]$ \\
\hline 28 & 3.7 & 30 & 19.5 & 5.8 & 30 & $8.50[6.04,10.96]$ \\
\hline 25.4 & 4.9 & 46 & 14 & 11.6 & 46 & $11.40[7.76,15.04]$ \\
\hline 27.1 & 5.4 & 30 & 15.2 & 8.2 & 30 & $11.90[8.39,15.41]$ \\
\hline 27.5 & 4.6 & 131 & 13.5 & 11.9 & 131 & $14.00[11.82,16.18]$ \\
\hline
\end{tabular}

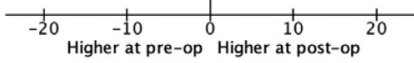

\begin{tabular}{l} 
Ctudy or Subgroup \\
\hline 3.3.1 Goniometer \\
Kelly et al 2012 \\
Riff et al 2018 \\
Carton \& Filan 2020 \\
Mullins et al 2020 \\
3.3.2 Not described \\
Flores et al 2018 (Early) \\
Nawabi et al 2016 (BD) \\
Flores et al 2018 (Late) \\
Nawabi et al 2016 (No BD) \\
Frank et al 2018 (Yoga) \\
Waterman et al 2018
\end{tabular}

Fig 3. Forest plot of studies reporting ROM in mixed (unilateral and bilateral) patients. The change in (A) flexion, (B) internal rotation, and (C) external rotation in studies including bilaterally operated patients following arthroscopic correction of femoroacetabular impingement (assessed using computed tomography simulation, goniometer and where exact technique not described) compared with preoperatively measured values. Overall heterogeneity (as assessed using $\mathrm{I}^{2}$ value) was $91 \%, 87 \%$ and $66 \%$ for flexion, internal rotation (IR) and external rotation (ER), respectively. (CI, confidence interval; IV, inverse variance; ROM, range of motion; SD, standard deviation.) of this bony deformity may also impact certain movements over others. A superiorly placed cam deformity has been shown to correlate with reduced ER, whereas a more anteriorly placed cam deformity correlates with a reduced IR. ${ }^{3}$ A superolateral cam lesion may impinge more in flexion and abduction. ${ }^{32}$ The size or presence of this bony deformity alone, however, may not be solely associated with an observed decrease in $\operatorname{ROM}^{14,17,50}$ and therefore overall structural anatomy should be considered. While morphology with respect to the acetabular side(pincer) is less well investigated for its influence on ROM, an association with lower abduction $^{12}$ has been reported. In the case of rim fractures, which are indicative of a more chronic and severe type of pincer impingement, adduction and IR have been shown to be significantly reduced. ${ }^{51}$

Surgical removal of the abnormal bone, verified and quantified by the change in the osseous angular measurements, should therefore result in a greater range of unobstructed hip movement, particularly hip flexion and IR. Although only a proportion of all studies included in this systematic review quantified the change in the osseous angular measurements from preto postoperative (13 reporting alpha angle changes; 8 reporting LCEA changes), in all of these studies there was significant improvements in these measurements following arthroscopic surgery. Similarly, among these studies, there was significant improvements in 
Table 3. Changes in Radiographic Measured Angles Pre- to Postoperatively

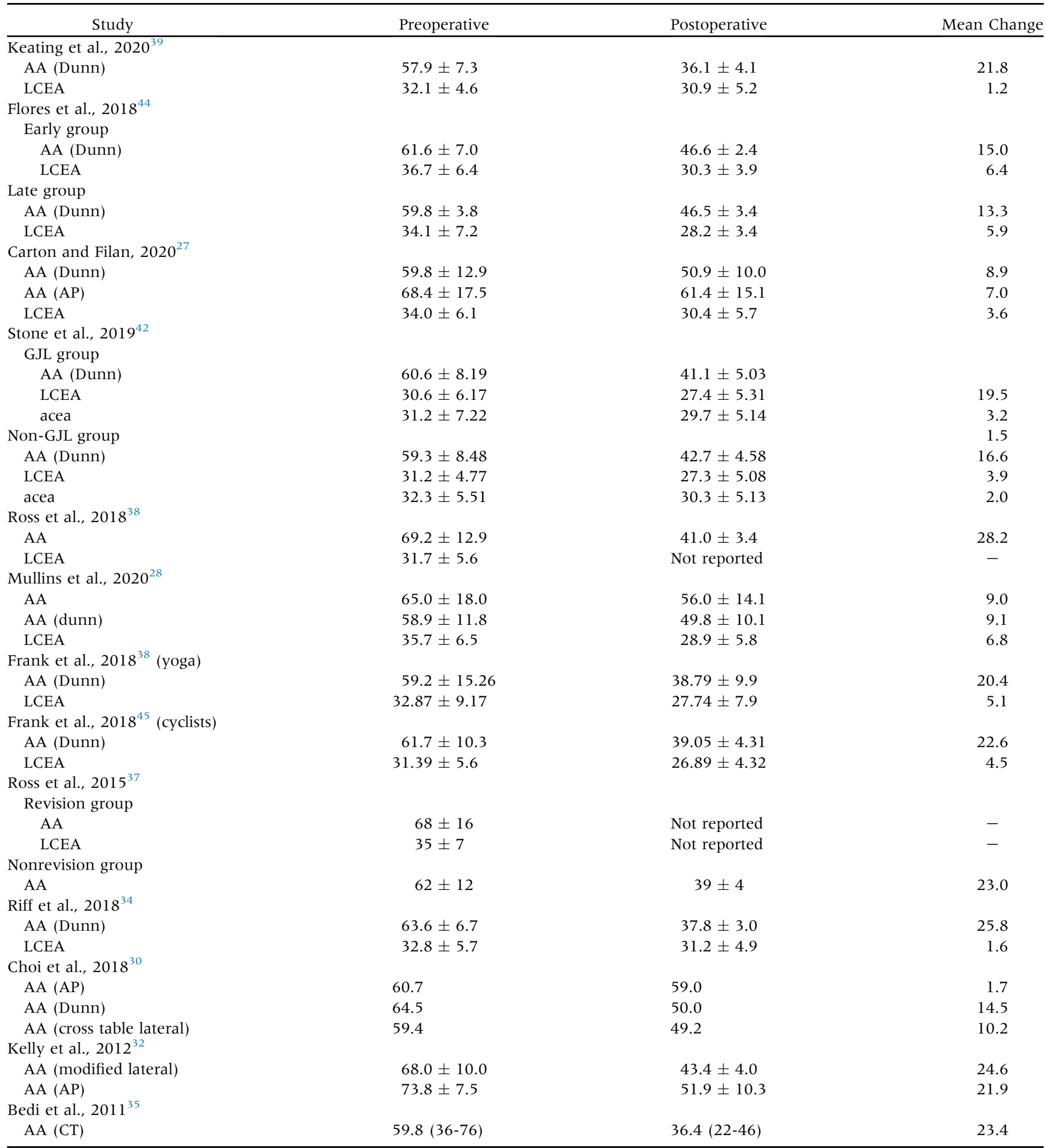

AA, alpha angle; ACEA, anterior center-edge angle; AP, anteroposterior view radiograph; CT, computed tomography; GJL, generalized joint laxity; LCEA, lateral center-edge angle.

measured ROMs from baseline to postoperative ( $100 \%$ of the studies evaluating IR; $76.9 \%$ of the studies evaluating flexion; $23 \%$ of the studies evaluating ER). Despite this, the association between the extent of resection with any changes in ROM was not wholly explored. For the majority, changes in ROM from baseline were largely reported as an incidental and accompanying outcome, and not the major focus of 
each included study. Without the authors assessing for a relationship between these 2 variables (ROM and bony resection) it cannot be concluded that resection alone impacted the change in ROM, however it is reasonable to assume a connection. For example, Kelly et al. ${ }^{32}$ did investigate this and reported that a change in alpha angle correlated with magnitude of increase in IR measured immediately after cam decompression $(\mathrm{r}=0.35)$ supporting a link between these 2 variables. In this same study, however, flexion was reported to be not significantly improved immediately following cam decompression; however, there was a significant improvement in flexion when this measurement was repeated at 3-months postoperatively. On the one hand, this may hint at factors beyond bony correction influencing this particular ROM; however, it also may suggest inconsistencies in measurement technique between the 2 time points. The time point at which ROM is assessed postoperatively may also influence the acquired measurable change in ROM. For example, Choi et al. ${ }^{30}$ longitudinally assessed flexion, IR and ER at 3 months, 6 months, 1 year, and 2 years' postoperatively and compared time-related measured values with baseline scores. While the association of bony resection with any changes to ROM was not evaluated, the time point at which significant improvement in ROM was achieved varied: IR by 3 months and ER by 6 months, whereas flexion did not significantly increase at any stage postoperatively.

Stähelin et al. ${ }^{47}$ reported that neither postoperative alpha value nor difference in alpha value achieved by correction (average $21.3^{\circ}$ ) correlated with any clinical parameters, despite significant increases postoperatively for IR and flexion reported. In this early study as part of the surgical technique the authors described a "large opening of the capsule," which was not reported to have been repaired. In this instance, therefore, the unrepaired capsulotomy may have, in part, contributed to the observed increase in IR and flexion. Subsequent research has since demonstrated an associated lack of restraint with an unrepaired capsule and therefore increased ROM following capsulotomy. ${ }^{52-54}$ In predisposed stiff hips, a capsulotomy may be therapeutic. ${ }^{55}$ Conversely, an overzealous capsular plication may constrain hip motion but may be potentially warranted in certain demographic instances. In the included study by Frank et al., ${ }^{38}$ assessing patients involved in yoga, an activity where patients typically exceed the physiologic joint tolerance, the authors state capsular plication may be critical to enhance joint kinematics while maintaining stability. As such, while the fundamentals of arthroscopic correction of cam and pincer deformities aim to restore joint mechanics to a more optimal physiologic state by increasing the available ROM in the typical FAI candidate, subsequent management of the capsule may also dictate the extent of available ROM postoperatively.

Overall bony anatomy beyond isolated cam and/or pincer deformities should be considered for their influence on restricted ROM. Two studies in this systematic review ${ }^{31,32}$ evaluated the impact of femoral version on hip ROM using goniometric measurements. Fabricant et al. $^{31}$ reported a greater postoperative change in IR in a decreased version group; however, this difference was determined to be largely owing to the significantly lower measured preoperative IR compared with normal and increased version groups. No differences in postoperative improvements in flexion or ER between version groups were observed. Similarly, Kelly et al. ${ }^{32}$ reported improved IR in all version groups following arthroscopic cam decompression, with a change in alpha angle correlating with magnitude of increase in IR. Another study ${ }^{41}$ compared the arthroscopic treatment of FAI among patients with borderline dysplasia versus nondysplasia and found there to be no significant differences in flexion, IR, or ER between groups at any time point. The significance of the change from preoperative to postoperative was not reported; however, both groups similarly trended with an increase in the measured mean IR and ER and a decrease in the measured flexion from baseline to postoperative. Natural structural differences (including decreased femoral anteversion, ${ }^{56}$ increased acetabular retroversion, ${ }^{14}$ anterior pelvic tilt, coxa vara/valga, or prominence of the anterior inferior iliac spine resulting from traction hypertrophy during adolescent development, ${ }^{57}$ etc.) may restrict hip rotation through various planes and result in variations of baseline measurements between patients, an important consideration when making cross-comparisons between studies. However, these natural structural variations are not addressed with typical FAI-corrective arthroscopic surgery and therefore their presence is independent of any acquired ROM change postarthroscopy.

Additional structural components, considered barriers to movement, may influence the measurement to endrange, such as surrounding periarticular soft tissues, capsule, cartilage, labrum, and muscles and based on the particular technique with which ROM is assessed, these structures may lead to either an under- or overestimation of functional range. Historically, the modality through which hip joint ROM has to be measured is variable, including use of goniometer, inclinometer, photometer, radiographs, and video tracking. ${ }^{58,59}$ Within this systematic review the predominantly reported ROM measurement techniques were goniometer and CT simulation. Where CT-simulation studies provide an understanding of the ROM in terms of boneto-bone available range, such studies ignore the aforementioned secondary stabilizing structures and may 
overestimate the functional benefits gained from arthroscopic correction for the patient. Goniometric evaluation is more appropriate in a clinical setting. ${ }^{60}$ The goniometer has demonstrated excellent intrarater reliability even in the unskilled examiner (intraclass correlation coefficient $0.906, P<.05)^{61}$ and good-toexcellent intra-rater, test-retest reliability for measuring hip flexion ROM. ${ }^{62}$ Of note, Nussbaumer et al. ${ }^{63}$ has shown some overestimation of hip ROM with the use of goniometer versus an electromagnetic tracking system.

Within interventional outcome studies, a number of PROs have been developed and validated to capture and quantify perceived change following arthroscopic correction of FAI and a description of these are generally required to be defined within a study's methodology. Similarly, when reporting the outcomes of surgical intervention of any type, a prerequisite is a comprehensive description of the surgical intervention technique with which the results have been conceived. Upon reviewing the literature retrieved during the search process, it is apparent that surgical outcome studies were overall lacking in their reporting of ROM changes following surgical correction of FAI. In particular, the sustainability of any acquired change over time in the form of longer-term outcome research is in stark contrast to the vast body of research evaluating outcomes from the perspective of PROs. An observation within this systematic review is the lack of any standardized or even descriptive "per-study" measurement protocol for clinically assessing ROM. Only $48 \%$ of the included studies reported the instrument with which ROM was measured, and even fewer detailing the technique. As such, guidance for repeatability of reported results in a clinical setting is inconsistent and poorly defined within the clinical outcome studies, which impacts the generalizability and direct comparability between cohorts.

The factors that have led to an under-reporting of ROM change over time is unclear. Considering these data can only be accurately captured via a third-party assessor (not the patient themselves), it can be assumed that logistics and practicality for a patient to return to a clinical setting for full assessment may be a significant hurdle. Large hip registries, which are the source of data retrieval for the majority of HA outcome studies, may be particularly affected by this. Sansone et al. ${ }^{64}$ have previously commented on the revision of a Swedish hip arthroscopy registry to exclude ROM owing to the fact they found this to be unreliable. Further, as there is no standardized technique with which all clinicians measure ROM (1 vs 2 operators, patient positioning (supine, seated, prone), same assessor(s) at different time points, manual/electronic goniometer, standardized technique/protocol to control for anatomic or environmental variations which may contribute to inaccuracies in true end range measurement, etc.), comparisons across studies are therefore less reliable and valid. From a clinical perspective, any change in measurable compound movements may lead to a more subjective feeling of improvement for the patient and should be considered for future studies assessing clinical outcome of HA in these cohorts

\section{Limitations}

Retrospective design of the included studies may introduce selection bias. The predominance of studies of lover level of evidence, underlying fair methodologic design and unavailability of randomized controlled trials assessing the influence of arthroscopic treatment on ROM may introduce further bias. Although the search was carried out in a systematic way, it is possible that studies which do report comparative ROM values were missed. English-only language search is also a limitation. In addition, only $52 \%$ of the papers provided enough detailed information with respect to the technique used to measure ROM, which may have introduced heterogeneity in the study methods and subsequent outcomes. The inclusion of bilateral patients when assessing ROM may result in reported CIs to be artificially narrow owing to correlation between 2 hip measurements from the same patient. The followup duration and sample sizes between studies included were variable. Finally, given the available evidence, the current study only assessed the influence of HA on the three more commonly assessed ROMs in isolation.

\section{Conclusions}

Outcome studies demonstrate overall increased range of flexion and IR post-HA, with a moderate and large effect respectively. Change in ER is less impacted following HA. Certainty of evidence to support this observation is low. Current research evaluating change in this functional ability is limited by a lack of prospective studies and nonstandardized measurement evaluation techniques.

\section{References}

1. Byrd JWT. Evaluation of the hip: History and physical examination. N Am J Sports Phys Ther 2007;2:231-240.

2. Frangiamore S, Mannava S, Geeslin AG, Chahla J, Cinque ME, Philippon MJ. Comprehensive clinical evaluation of femoroacetabular impingement: Part 1, Physical examination. Arthrosc Tech 2017;6:e1993-e2001. doi:10. 1016/j.eats.2017.03.027.

3. Jónasson P, Thoreson O, Sansone M, et al. The morphologic characteristics and range of motion in the hips of athletes and non-athletes. J Hip Preserv Surg 2016;3: hnw023. doi:10.1093/jhps/hnw023.

4. Nieszporska O, Truszczyńska-Baszak A. Functional condition of patients after unilateral hip arthroscopy in the process of FAI-femoroacetabular impingement: A 
case-control study and preliminary report. J Clin Med 2021;10:1023. doi:10.3390/jcm10051023.

5. Philippon MJ, Briggs KK, Yen YM, Kuppersmith DA. Outcomes following hip arthroscopy for femoroacetabular impingement with associated chondrolabral dysfunction: Minimum two-year follow-up. J Bone Joint Surg $\mathrm{Br}$ 2009;91:16-23. doi:10.1302/0301-620X.91B1.21329.

6. Philippon MJ, Maxwell RB, Johnston TL, Schenker M, Briggs KK. Clinical presentation of femoroacetabular impingement. Knee Surg Sports Traumatol Arthrosc 2007;15: 1041-1047. doi:10.1007/s00167-007-0348-2.

7. Roach KE, Miles TP. Normal hip and knee active range of motion: The relationship to age. Phys Ther 1991;71: 656-665. doi:10.1093/ptj/71.9.656.

8. Agricola R, Heijboer MP, Ginai AZ, et al. A cam deformity is gradually acquired during skeletal maturation in adolescent and young male soccer players: A prospective study with minimum 2-year follow-up. Am J Sports Med 2014;42:798-806.

9. Anwander H, Beck M, Büchler L. Influence of evolution on cam deformity and its impact on biomechanics of the human hip joint. J Orthop Res 2018;36:2071-2075. doi:10. 1002 /jor.23863.

10. Kapron AL, Anderson AE, Peters CL, et al. Hip internal rotation is correlated to radiographic findings of cam femoroacetabular impingement in collegiate football players. Arthroscopy 2012;28:1661-1670. doi:10.1016/j. arthro.2012.04.153.

11. van Klij P, Ginai AZ, Heijboer MP, Verhaar JAN, Waarsing JH, Agricola R. The relationship between cam morphology and hip and groin symptoms and signs in young male football players. Scand J Med Sci Sports 2020;30:1221-1231. doi:10.1111/sms.13660.

12. Mosler AB, Agricola R, Thorborg K, et al. Is bony hip morphology associated with range of motion and strength in asymptomatic male soccer players? J Orthop Sports Phys Ther 2018;48:250-259. doi:10.2519/jospt.2018.7848.

13. Wyss TF, Clark JM, Weishaupt D, Nötzli HP. Correlation between internal rotation and bony anatomy in the hip. Clin Orthop Rel Res 2007;(460):152-158. doi:10.1097/BLO. Ob013e3180399430.

14. Audenaert EA, Peeters I, Vigneron L, Baelde N, Pattyn C. Hip morphological characteristics and range of internal rotation in femoroacetabular impingement. Am J Sports Med 2012;40:1329-1336. doi:10.1177/0363546512441328.

15. Frasson VB, Vaz MA, Morales AB, et al. Hip muscle weakness and reduced joint range of motion in patients with femoroacetabular impingement syndrome: A casecontrol study. Braz J Phys Ther 2020;24:39-45. doi:10. 1016/j.bjpt.2018.11.010.

16. Wörner T, Nilsson J, Thorborg K, Granlund V, Stålman A, Eek F. Hip function 6 to 10 months after arthroscopic surgery: A cross-sectional comparison of subjective and objective hip function, including performance-based measures, in patients versus controls. Orthop J Sports Med 2019;7:1-10. doi:10.1177/2325967119844821.

17. Freke MD, Kemp J, Svege I, Risberg MA, Semciw A, Crossley KM. Physical impairments in symptomatic femoroacetabular impingement: A systematic review of the evidence. Br J Sports Med 2016;50:1180. doi:10.1136/ bjsports-2016-096152.
18. Nwachukwu BU, Beck EC, Kunze KN, Chahla J, Rasio J, Nho SJ. Defining the clinically meaningful outcomes for arthroscopic treatment of femoroacetabular impingement syndrome at minimum 5-year follow-up. Am J Sports Med 2020;48:901-907. doi:10.1177/0363546520902736.

19. Zimmerer A, Janz V, Sobau C, Wassilew GI, Miehlke W. Defining the clinically meaningful outcomes for arthroscopic treatment of femoroacetabular impingement syndrome at minimum 10-year follow-up: The timing of surgery is crucial. Orthop J Sports Med 2021;9:4-11. doi:10. $1177 / 2325967120985140$.

20. Page MJ, McKenzie JE, Bossuyt PM, et al. The PRISMA 2020 statement: An updated guideline for reporting systematic reviews. BMJ 2021:372. doi:10.1136/bmj.n71.

21. Slim K, Nini E, Forestier D, Kwiatkowski F, Panis Y, Chipponi J. Methodological index for non-randomized studies (Minors): Development and validation of a new instrument. ANZ J Surg 2003;73:712-716. doi:10.1046/j. 1445-2197.2003.02748.x.

22. Sundemo D, Hamrin Senorski E, Karlsson L, et al. Generalised joint hypermobility increases ACL injury risk and is associated with inferior outcome after ACL reconstruction: A systematic review. BMJ Open Sport Exerc Med 2019;5. doi:10.1136/bmjsem-2019-000620.

23. Hozo SP, Djulbegovic B, Hozo I. Estimating the mean and variance from the median, range, and the size of a sample. BMC Med Res Methodol 2005;5:1-10. doi:10.1186/14712288-5-13.

24. Griffin DW, Kinnard MJ, Formby PM, McCabe MP, Anderson TD. Outcomes of hip arthroscopy in the older adult: A systematic review of the literature. Am J Sports Med 2017;45:1928-1936. doi:10.1177/0363546516667915.

25. Di Benedetto P, Barbattini P, Povegliano L, et al. Extracapsular vs standard approach in hip arthroscopy. Acta Biomed 2016:87:41-45.

26. Hohmann E, Feldman M, Hunt TJ, Cote MP, Brand JC. Research pearls: How do we establish the level of evidence? Arthroscopy 2018;34:3271-3277. doi:10.1016/J. ARTHRO.2018.10.002.

27. Carton P, Filan D. Defining the minimal clinically important difference in athletes undergoing arthroscopic correction of sports-related femoroacetabular impingement: The percentage of possible improvement. Orthop $J$ Sports Med 2020. doi:10.1177/2325967119894747:81-10.

28. Mullins K, Hanlon M, Carton P. Arthroscopic correction of femoroacetabular impingement improves athletic performance in male athletes. Knee Surg Sports Traumatol Arthrosc 2020;28:2285-2294. doi:10.1007/s00167-01905683-0.

29. Stone AV, Mehta N, Beck EC, et al. Comparable patientreported outcomes in females with or without joint hypermobility after hip arthroscopy and capsular plication for femoroacetabular impingement syndrome. J Hip Preserv Surg 2019;6:33-40. doi:10.1093/jhps/hnz004.

30. Choi SM, Park MS, Ju BC, Yoon SJ. Alterations in range of motion and clinical outcomes after femoroplasty in Asians. J Am Acad Orthop Surg 2018;26:e181-e190. doi:10. 5435/JAAOS-D-16-00944.

31. Fabricant PD, Fields KG, Taylor SA, Magennis E, Bedi A, Kelly BT. The effect of femoral and acetabular version on clinical outcomes after arthroscopic femoroacetabular 
impingement surgery. J Bone Joint Surg Am 2015;97: 537-543. doi:10.2106/JBJS.N.00266.

32. Kelly BT, Bedi A, Robertson CM, Dela Torre K, Giveans MR, Larson CM. Alterations in internal rotation and alpha angles are associated with arthroscopic cam decompression in the hip. Am J Sports Med 2012;40: 1107-1112. doi:10.1177/0363546512437731.

33. Matsuda DK, Schnieder CP, Sehgal B. The critical corner of cam femoroacetabular impingement: Clinical support of an emerging concept. Arthroscopy 2014;30:575-580. doi: 10.1016/j.arthro.2014.01.009.

34. Riff AJ, Ukwuani G, Clapp I, Movassaghi K, Kelly DM, Nho SJ. High rate of return to high-intensity interval training after arthroscopic management of femoroacetabular impingement syndrome. Am J Sports Med 2018;46:2594-2600. doi:10.1177/0363546518776638.

35. Bedi A, Dolan M, Hetsroni I, et al. Surgical treatment of femoroacetabular impingement improves hip kinematics: A computer-assisted model. Am J Sports Med $2011 ; 39$ (1_suppl):43S-49S. doi:10.1177/ 0363546511414635.

36. Ross JR, Khan M, Noonan BC, Larson CM, Kelly BT, Bedi A. Characterization and correction of symptomatic hip impingement in American football linemen. HSS $J$ 2018;14:128-133. doi:10.1007/s1 1420-018-9605-9.

37. Ross JR, Larson CM, Adeoyo O, Kelly BT, Bedi A. Residual deformity is the most common reason for revision hip arthroscopy: A three-dimensional CT study. Clin Orthop Rel Res 2015;473:1388-1395. doi:10.1007/s11999014-4069-9.

38. Frank RM, Ukwuani G, Allison B, Clapp I, Nho SJ. High rate of return to yoga for athletes after hip arthroscopy for femoroacetabular impingement syndrome. Sports Health 2018;10:434-440. doi:10.1177/1941738118757406.

39. Keating TC, Chahla J, Beck EC, et al. Return to Pilates following hip arthroscopy for treatment of femoroacetabular impingement syndrome. J Hip Preserv Surg 2019;6:339-345. doi:10.1093/jhps/hnz053.

40. Levy DM, Cvetanovich GL, Kuhns BD, Greenberg MJ, Alter JM, Nho SJ. Hip arthroscopy for atypical posterior hip pain: A comparative matched-pair analysis. Am J Sports Med 2017;45:1627-1632. doi:10.1177/0363546517692983.

41. Nawabi DH, Degen RM, Fields KG, et al. Outcomes after arthroscopic treatment of femoroacetabular impingement for patients with borderline hip dysplasia. Am J Sports Med 2016;44:1017-1023. doi:10.1177/0363546515624682.

42. Stone AV, Malloy P, Beck EC, et al. Predictors of persistent postoperative pain at minimum 2 years after arthroscopic treatment of femoroacetabular impingement. Am J Sports Med 2019;47:552-559. doi:10.1177/0363546518817538.

43. Waterman BR, Ukwuani G, Clapp I, Malloy P, Neal WH, Nho SJ. Return to golf after arthroscopic management of femoroacetabular impingement syndrome. Arthroscopy 2018;34:3187-3193.el. doi:10.1016/j.arthro.2018.06.042.

44. Flores SE, Borak KR, Zhang AL. Hip arthroscopic surgery for femoroacetabular impingement: A prospective analysis of the relationship between surgeon experience and patient outcomes. Orthop J Sports Med 2018;6:1-8. doi:10. $1177 / 2325967118755048$.

45. Frank RM, Ukwuani G, Clapp I, Chahla J, Nho SJ. High rate of return to cycling after hip arthroscopy for femoroacetabular impingement syndrome. Sports Health 2018;10:259-265. doi:10.1177/1941738117747851.

46. Ragab R, Elkhadrawe T, Housden P, Abotaleb A. Results of arthroscopic treatment of femoroacetabular impingement (FAI). Alexandria J Med 2018;54:361-363. doi:10. 1016/j.ajme.2018.04.002.

47. Stähelin L, Stähelin T, Jolles BM, Herzog RF. Arthroscopic offset restoration in femoroacetabular cam impingement: Accuracy and early clinical outcome. Arthroscopy 2008;24: 51-57.el. doi:10.1016/j.arthro.2007.08.010.

48. Polesello GC, Queiroz MC, Ono NK, Honda EK, Guimarāes RP, Junior WR. Arthroscopic treatment of femoroacetabular impingement. Rev Bras Ortop 2009;44: 230-238.

49. Guler O, Isyar M, Karataş D, Ormeci T, Cerci H, Mahirogullar M. A retrospective analysis on the correlation between hip pain, physical examination findings, and alpha angle on MR images. J Orthop Surg Res 2016;11:1-5. doi:10.1186/s13018-016-0476-9.

50. Tak I, Glasgow P, Langhout R, Weir A, Kerkhoffs G, Agricola R. Hip range of motion is lower in professional soccer players with hip and groin symptoms or previous injuries, independent of cam deformities. Am J Sports Med 2016;44:682-688. doi:10.1177/0363546515617747.

51. Carton PF, Filan DJ. The clinical presentation, diagnosis and pathogenesis of symptomatic sports-related femoroacetabular impingement (SRFAI) in a consecutive series of 1021 athletic hips. Hip Int 2019;29:665-673. doi:10. $1177 / 1120700018825430$.

52. Abrams GD, Hart MA, Takami K, et al. Biomechanical evaluation of capsulotomy, capsulectomy, and capsular repair on hip rotation. Arthroscopy 2015;31:1511-1517. doi:10.1016/j.arthro.2015.02.031.

53. Bayne CO, Stanley R, Simon P, et al. Effect of capsulotomy on hip stability - a consideration during hip arthroscopy. Am J Orthop (Belle Mead NJ) 2014;43: $160-165$.

54. Filan D, Carton P. Routine interportal capsular repair does not lead to superior clinical outcome following arthroscopic femoroacetabular impingement correction with labral repair. Arthroscopy 2020;36:1323-1334. doi:10. 1016/j.arthro.2019.12.002.

55. Harris JD, Slikker W, Gupta AK, McCormick FM, Nho SJ. Routine complete capsular closure during hip arthroscopy. Arthrosc Tech 2013;2:e89-e94. doi:10.1016/j.eats. 2012.11.007.

56. Ejnisman L, Philippon MJ, Lertwanich P, et al. Relationship between femoral anteversion and findings in hips with femoroacetabular impingement. Orthopedics 2013;36: 293-300. doi:10.3928/01477447-20130222-17.

57. Carton P, Filan D. Anterior inferior iliac spine (AIIS) and subspine hip impingement. Muscles Ligaments Tendons $J$ 2016;6:324-336. doi:10.11138/mltj/2016.6.3.324.

58. Kouyoumdjian P, Coulomb R, Sanchez T, Asencio G. Clinical evaluation of hip joint rotation range of motion in adults. Orthop Traumatol Surg Res 2012;98:17-23. doi:10. 1016/j.otsr.2011.08.015.

59. Yazdifar M, Yazdifar MR, Mahmud J, Esat I, Chizari M. Evaluating the hip range of motion using the goniometer and video tracking methods. Proced Engin 2013;68:77-82. doi:10.1016/j.proeng.2013.12.150. 
60. Prather H, Harris-Hayes M, Hunt D, Steger-May K, Mathew V, Clohisy JC. Reliability and agreement of hip range of motion and provocative physical examination tests in asymptomatic volunteers. PM R 2010;2:888-895. doi:10.1016/j.pmrj.2010.05.005. Hip.

61. Kim SG, Kim EK. Test-retest reliability of an active range of motion test for the shoulder and hip joints by unskilled examiners using a manual goniometer. J Phys Ther Sci 2016;28:722-724. doi:10.1589/jpts.28.722.

62. Pua YH, Wrigley TW, Cowan SM, Bennell KL. Intrarater test-retest reliability of hip range of motion and hip muscle strength measurements in persons with hip osteoarthritis. Arch Phys Med Rehab 2008;89:1146-1154. doi:10.1016/j.apmr.2007.10.028.

63. Nussbaumer S, Leunig M, Glatthorn JF, Stauffacher S, Gerber H, Maffiuletti NA. Validity and test-retest reliability of manual goniometers for measuring passive hip range of motion in femoroacetabular impingement patients. BMC Musculoskelet Disord 2010;1 1:194. doi:10.1186/ 1471-2474-11-194.

64. Sansone M, Ahldén M, Jonasson P, et al. A Swedish hip arthroscopy registry: Demographics and development. Knee Surg Sports Traumatol Arthrosc 2014;22:774-780. doi: 10.1007/s00167-014-2840-9. 
Appendix Table 1. Search Terms Used

$(((($ (femoroacetabular") OR ("femoro acetabular")) OR ("femoro-acetabular")) AND (("impingement") OR ("impingement syndrome"))) AND (("hip arthroscopy") OR ("arthroscopic correction"))) AND $((()((()(()(()(()((()$ "hip range of motion") OR (range of motion)) OR (range of motion, articular)) OR ("range of motion")) OR ("range of movement")) OR ("ROM")) OR ("flexion")) OR ("abduction")) OR ("adduction")) OR ("external rotation")) OR ("internal rotation")) OR (flex*)) OR (abduct*)) OR (adduct*)) OR (exter*)) OR (intern*)) OR ("extension")) OR $($ exten*)) OR ("squat")) OR ("depth")) OR (rotat*)) 


\begin{tabular}{|c|c|c|c|c|c|c|c|c|c|c|c|c|c|c|}
\hline & \multicolumn{8}{|c|}{ Non-Comparative (/16) } & \multicolumn{4}{|c|}{ Comparative (/24) } & \multirow[b]{2}{*}{$\begin{array}{c}\text { Total } \\
\text { MINORS } \\
\text { Score }\end{array}$} & \multirow[b]{2}{*}{$\begin{array}{l}\text { Study } \\
\text { Quality }\end{array}$} \\
\hline & $\begin{array}{l}\text { A clearly } \\
\text { stated } \\
\text { aim } \\
\end{array}$ & $\begin{array}{c}\text { Inclusion of } \\
\text { consecutive } \\
\text { patients }\end{array}$ & $\begin{array}{c}\text { Prospective } \\
\text { collection } \\
\text { of data }\end{array}$ & $\begin{array}{l}\text { Endpoints } \\
\text { appropriate } \\
\text { to aim of } \\
\text { the study }\end{array}$ & $\begin{array}{c}\text { Unbiased } \\
\text { assessment of } \\
\text { the study } \\
\text { endpoint }\end{array}$ & $\begin{array}{l}\text { Follow-up } \\
\text { period } \\
\text { f appropriate to } \\
\text { aim of } \\
\text { study } \\
\end{array}$ & $\begin{array}{c}\text { Loss of } \\
\text { follow-up } \\
\text { less } \\
\text { than } 5 \% \\
\end{array}$ & $\begin{array}{l}\text { Prospective } \\
\text { calculation of } \\
\text { the study } \\
\text { size }\end{array}$ & $\begin{array}{c}\text { An } \\
\text { adequate } \\
\text { control } \\
\text { group } \\
\end{array}$ & $\begin{array}{c}\text { Contemporary } \\
\text { groups }\end{array}$ & $\begin{array}{c}\text { Baseline } \\
\text { equivalence } \\
\text { of groups }\end{array}$ & $\begin{array}{c}\text { Adequate } \\
\text { statistical } \\
\text { analysis } \\
\end{array}$ & & \\
\hline Keating et al. & 2 & 2 & 1 & 2 & 0 & 2 & 1 & 0 & & & & & 10 & Fair \\
\hline Ragab et al. & 1 & 0 & 0 & 2 & 0 & 1 & 0 & 0 & & & & & 4 & Very low \\
\hline $\begin{array}{l}\text { Waterman } \\
\text { et al. }\end{array}$ & 2 & 2 & 1 & 2 & 0 & 2 & 1 & 0 & & & & & 10 & Fair \\
\hline Flores et al. & 2 & 2 & 2 & 2 & 0 & 1 & 2 & 2 & & & & & 13 & High \\
\hline $\begin{array}{l}\text { Carton and } \\
\text { Filan }\end{array}$ & 2 & 1 & 2 & 2 & 0 & 2 & 1 & 0 & & & & & 10 & Fair \\
\hline Stone et al. & 2 & 0 & 2 & 2 & 0 & 2 & 0 & 2 & 2 & 1 & 2 & 2 & 17 & Fair \\
\hline Ross et al. & 2 & 2 & 1 & 2 & 0 & 2 & 2 & 0 & & & & & 11 & Fair \\
\hline $\begin{array}{l}\text { Polesello } \\
\text { et al. }\end{array}$ & 2 & 0 & 0 & 1 & 0 & 2 & 2 & 0 & & & & & 7 & Low \\
\hline Mullins et al. & 2 & 0 & 2 & 2 & 0 & 2 & 1 & 2 & 2 & 2 & 2 & 2 & 19 & High \\
\hline Stone et al. & 2 & 2 & 2 & 2 & 0 & 2 & 1 & 0 & 1 & 2 & 1 & 2 & 17 & Fair \\
\hline Frank et al. & 2 & 2 & 2 & 2 & 0 & 2 & 2 & 0 & & & & & 12 & Fair \\
\hline Frank et al. & 2 & 2 & 2 & 2 & 0 & 2 & 1 & 0 & & & & & 11 & Fair \\
\hline Levy et al. & 2 & 2 & 0 & 2 & 0 & 2 & 1 & 0 & 2 & 2 & 2 & 2 & 17 & Fair \\
\hline Nawabi et al. & 2 & 2 & 2 & 2 & 0 & 2 & 0 & 0 & 2 & 2 & 1 & 2 & 17 & Fair \\
\hline $\begin{array}{l}\text { Fabricant } \\
\text { et al. }\end{array}$ & 2 & 2 & 2 & 2 & 0 & 2 & 2 & 0 & & & & & 12 & Fair \\
\hline Ross et al. & 2 & 2 & 0 & 2 & 0 & 2 & 2 & 0 & & & & & 10 & Fair \\
\hline Stähelin et al. & 2 & 2 & 2 & 1 & 0 & 1 & 2 & 0 & & & & & 10 & Fair \\
\hline Riff et al. & 2 & 2 & 2 & 2 & 0 & 2 & 2 & 1 & & & & & 13 & High \\
\hline $\begin{array}{l}\text { Matsuda } \\
\text { et al. }\end{array}$ & 2 & 2 & 0 & 2 & 0 & 2 & 2 & 0 & & & & & 10 & Fair \\
\hline Choi et al. & 2 & 2 & 0 & 2 & 0 & 2 & 2 & 0 & & & & & 10 & Fair \\
\hline Kelly et al. & 2 & 2 & 0 & 2 & 0 & 2 & 2 & 0 & & & & & 10 & Fair \\
\hline Bedi et al. & 2 & 0 & 0 & 2 & 0 & 2 & 2 & 0 & & & & & 8 & Fair \\
\hline $\begin{array}{l}\text { Di Benedetto } \\
\text { et al. }\end{array}$ & 1 & 2 & 0 & 2 & 0 & 1 & 0 & 0 & & & & & 6 & Low \\
\hline
\end{tabular}




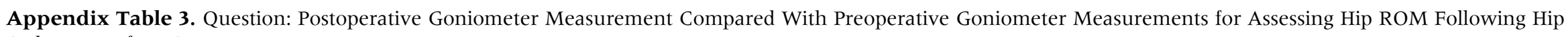
Arthroscopy for FAI

\begin{tabular}{|c|c|c|c|c|c|c|c|c|c|c|c|c|}
\hline \multicolumn{7}{|c|}{ Certainty Assessment } & \multicolumn{2}{|c|}{ No. of Patients } & \multicolumn{2}{|r|}{ Effect } & \multirow[b]{2}{*}{ Certainty } & \multirow[b]{2}{*}{ Importance } \\
\hline $\begin{array}{c}\text { No. of } \\
\text { Studies }\end{array}$ & $\begin{array}{l}\text { Study } \\
\text { Design }\end{array}$ & $\begin{array}{l}\text { Risk of } \\
\text { Bias }\end{array}$ & Inconsistency & Indirectness & Imprecision & $\begin{array}{c}\text { Other } \\
\text { Considerations }\end{array}$ & $\begin{array}{c}\text { Postoperative } \\
\text { Goniometer } \\
\text { Measurement }\end{array}$ & $\begin{array}{c}\text { Preoperative } \\
\text { Goniometer } \\
\text { Measurements }\end{array}$ & $\begin{array}{l}\text { Relative } \\
(95 \% \mathrm{CI}) \\
\end{array}$ & $\begin{array}{l}\text { Absolute } \\
(95 \% \text { CI }) \\
\end{array}$ & & \\
\hline \multicolumn{13}{|c|}{ Flexion_Goniometer } \\
\hline 6 & $\begin{array}{l}\text { Observational } \\
\text { studies }\end{array}$ & Serious* & Serious & Not serious & Not serious & $\begin{array}{l}\text { Publication bias } \\
\text { strongly } \\
\text { suspected }\end{array}$ & 748 & 748 & - & $\begin{array}{l}\text { MD } 5.98 \text { higher } \\
\text { (2.99 higher to } \\
8.98 \text { higher) }\end{array}$ & $\begin{array}{c}\oplus \bigcirc \bigcirc \bigcirc \\
\text { VERY } \\
\text { LOW }\end{array}$ & IMPORTAN \\
\hline \multicolumn{13}{|c|}{ Internal Rotation_Goniometer } \\
\hline 8 & $\begin{array}{l}\text { observational } \\
\text { studies }\end{array}$ & Serious* & Serious* & Not serious & Not serious & $\begin{array}{l}\text { Publication bias } \\
\text { strongly } \\
\text { suspected }\end{array}$ & 787 & 787 & - & $\begin{array}{c}\text { MD } 11.68 \text { higher } \\
\text { (8.13 higher to } \\
15.23 \text { higher) }\end{array}$ & $\begin{array}{c}\oplus O \bigcirc \bigcirc \\
\text { VERY } \\
\text { LOW }\end{array}$ & IMPORTAN \\
\hline \multicolumn{13}{|c|}{ External Rotation_Goniometer } \\
\hline 6 & $\begin{array}{l}\text { Observational } \\
\text { studies }\end{array}$ & Serious* & Serious ${ }^{*}$ & Not serious & Not serious & $\begin{array}{l}\text { Publication bias } \\
\text { strongly } \\
\text { suspected }\end{array}$ & 748 & 748 & - & $\begin{array}{c}\text { MD } 2.68 \text { higher } \\
\text { (1.21 lower to } \\
6.56 \text { higher })\end{array}$ & $\begin{array}{c}\oplus \text { OO० } \\
\text { VERY } \\
\text { LOW }\end{array}$ & IMPORTAN \\
\hline
\end{tabular}

CI, confidence interval; FAI, femoroacetabular impingement; MD, mean difference; ROM, range of motion.

*Retrospective study designs, measurement techniques not fully described and variation across studies, examiner not blinded. 
Appendix Table 4. Question: Postoperative CT Simulation Compared With Preoperative CT Simulation for Assessing Hip ROM Following Hip Arthroscopy for FAI

\begin{tabular}{|c|c|c|c|c|c|c|c|c|c|c|c|c|}
\hline \multicolumn{7}{|c|}{ Certainty Assessment } & \multicolumn{2}{|c|}{ No. of Patients } & \multicolumn{2}{|c|}{ Effect } & \multirow[b]{2}{*}{ Certainty } & \multirow[b]{2}{*}{ Importance } \\
\hline $\begin{array}{l}\text { No. of } \\
\text { Studies }\end{array}$ & $\begin{array}{l}\text { Study } \\
\text { Design }\end{array}$ & Risk of Bias & Inconsistency & Indirectness & Imprecision & $\begin{array}{c}\text { Other } \\
\text { Considerations }\end{array}$ & $\begin{array}{l}\text { Postoperative CT } \\
\text { Simulation }\end{array}$ & $\begin{array}{l}\text { Preoperative CT } \\
\text { Simulation }\end{array}$ & $\begin{array}{l}\text { Relative } \\
(95 \% \text { CI })\end{array}$ & $\begin{array}{l}\text { Absolute } \\
(95 \% \text { CI) }\end{array}$ & & \\
\hline \multicolumn{13}{|c|}{ Flexion_All Studies - Simulated } \\
\hline 3 & Observational studies & Not serious & Not serious & Not serious & Not serious & None & 92 & 92 & - & $\begin{array}{l}\text { MD } 7.28 \\
\text { higher }(4.1 \\
\text { higher to } \\
10.45 \\
\text { higher) }\end{array}$ & $\begin{array}{c}\oplus \oplus \bigcirc \bigcirc \\
\text { LOW }\end{array}$ & IMPORTANT \\
\hline \multicolumn{13}{|c|}{ Internal Rotation_All Studies. - Simulated } \\
\hline 3 & Observational studies & Not serious & Not serious & Not serious & Not serious & None & 92 & 92 & - & $\begin{array}{l}\text { MD } 13.22 \\
\text { higher } \\
(9.54 \\
\text { higher to } \\
16.9 \\
\text { higher) }\end{array}$ & $\begin{array}{c}\oplus \oplus \bigcirc \bigcirc \\
\text { LOW }\end{array}$ & IMPORTANT \\
\hline
\end{tabular}

CI, confidence interval; FAI, femoroacetabular impingement; MD, mean difference; ROM, range of motion. 